\title{
Controlling the magnetism of adsorbed metal-organic molecules
}

\author{
Wolfgang Kuch and Matthias Bernien \\ Institut für Experimentalphysik, Freie Universität Berlin, Arnimallee 14, 14195 \\ Berlin, Germany \\ E-mail: kuch@physik.fu-berlin.de
}

10. October 2016

\begin{abstract}
Gaining control on the size or the direction of the magnetic moment of adsorbed metal-organic molecules constitutes an important step towards the realization of a surface-mounted molecular spin electronics. Such control can be gained by taking advantage of interactions of the molecule's magnetic moment with the environment. The paramagnetic moments of adsorbed metal-organic molecules, for example, can be controlled by the interaction with magnetically ordered substrates. Metalloporphyrins and -phthalocyanines display a quasi-planar geometry, allowing the central metal ion to interact with substrate electronic states. This can lead to magnetic coupling with a ferromagnetic or even antiferromagnetic substrate. The molecule-substrate coupling can be mediated and controlled by insertion layers such as oxygen atoms, graphene, or nonmagnetic metal layers. Control on the magnetic properties of adsorbed metalloporphyrins or -phthalocyanines can also be gained by on-surface chemical modification of the molecules. The magnetic moment or the magnetic coupling to ferromagnetic substrates can be changed by adsorption and thermal desorption of small molecules that interact with the fourfold-coordinated metal center via the remaining axial coordination site. Spin-crossover molecules, which possess a metastable spin state that can be switched by external stimuli such as temperature or light, are another promising class of candidates for control of magnetic properties. However, the immobilization of such molecules on a solid surface often results in a quench of the spin transition due to the interaction with the substrate. We present examples of $\mathrm{Fe}(\mathrm{II})$ spin-crossover complexes in direct contact with a solid surface that undergo a reversible spin-crossover transition as a function of temperature, by illumination with visible light, or can be switched by the tip of a scanning tunneling microscope.
\end{abstract}

Keywords: Magnetic molecules, X-ray absorption spectroscopy, Spin crossover molecules

Submitted to: J. Phys.: Condens. Matter 


\section{Introduction}

The vision of using organic molecules as ultimately small building blocks in a future nanoelectronics instead of lithographic semiconductor structures has triggered an enormous amount of research work. The idea is to rebuild logic functions by assemblies of molecules. These could be logic operations to process information or the storage of information. Molecules have certain advantages over other materials. They can be produced in large quantities with exact reproducibility. Their functionality can be changed in a controlled way by chemical synthesis, for example by subtle modifications of ligands or other parts of the molecules. Furthermore, different functionalities may be added to the same molecule by different ligands.

If molecules possessing a magnetic moment are included in such a molecular electronics, spin-dependent transport phenomena might be within reach. This has been coined "molecular spintronics", in analogy to conventional spintronics in which magnetic materials are embedded in lithographic heterostructures to take advantage of the electron spin as carrier of information rather than its charge $[1,2,3,4]$. A major challenge in molecular spintronics is the stabilization of the magnetic moment of molecular building blocks against thermal fluctuations. Since the magnetic moments of individual molecules are rather small, thermal energy is usually surpassing the typical magnetic energies even at temperatures way below ambient temperatures, leading to the disappearance of the thermal average of the magnetic moment and thus to the disappearance of any spin-dependent effects. A second major challenge is the immobilization of suitable molecules, which is necessary for any kind of addressing or contacting. Over time, coordination chemistry has led to quite a number of molecules with exciting properties; however, once immobilized on a solid surface by adsorption, the molecule-surface interaction has a dominant influence on the electronic and magnetic properties of the molecule, and a quenching of the desired functionality is not at all uncommon.

Here is where surface science comes into play. Over the decades both experimental and theoretical progress have helped to understand also relatively complex adsorbatesurface systems. Although single crystals will probably not be part of future applications, single-crystalline surfaces are advantageous in experiments aimed at gaining fundamental insight into the processes governing the molecule-substrate interaction and their influence on the desired function.

To be able to perform logic operations, the control of molecular properties by the environment is required. A relatively straightforward way of controlling the magnetism of adsorbed molecules is to take advantage of magnetic coupling between the magnetic ion of an adsorbed molecule and a ferromagnetic substrate. Up to now, different mechanisms mediating such a coupling have been identified. They will be reviewed in section 2 .

For free molecules in gas phase or in solution, certain ways to control the molecular magnetic moment by means of external stimuli are known. Switching by chemical 
means, for example by attaching additional functional groups to a molecule, is one way. More sophisticated approaches have used photoisomerization to approach or retract a nitrogen-terminated ligand to the magnetic ion, resulting in spin state switching [5]. On surfaces, the flexibility and mobility of adsorbed molecules is restricted, and the same kind of switching has not yet been obtained. However, several experiments with different systems have shown the principal feasibility of this approach. Adsorbing small molecules from the gas phase to already-adsorbed magnetic molecules can lead to a characteristic change of the magnetic properties. In many cases the coadsorbed molecules can be desorbed in a reversible manner by increasing the substrate temperature, illustrating a path to a reversible switching of the molecular magnetism. The present state of such experiments will be presented in section 3.

Spin crossover (SCO) molecules are another example of a reversible modification of molecular magnetic properties. In the bulk phase or in solution, such molecules are known to change their magnetic moment by temperature, exposure to light, or pressure $[6,7]$. They would thus be a promising class of molecules for molecular spintronics, however, the delicate balance between spin-pairing energy and ligand field is readily disturbed by the adsorption on a solid surface. Quenching of the spin crossover transition of molecules in direct contact with a surface is most often the consequence. Recent experiments, however, demonstrated that for certain molecules, which can be evaporated in vacuum, and weakly interacting substrates such as graphite a complete spin-crossover switching by temperature and even by light is possible. This is the content of section 4 , in which we summarize the current state of such experiments.

\section{Controlling the paramagnetic moment by the interaction with magnetically ordered substrates}

Metalloporphyrins are planar molecules in which a single transition metal center ion is coordinated by four nitrogen atoms [8]. This allows to exert control on the ion's magnetic properties from the two remaining vertical coordination sites. Porphyrins typically adsorb flat on solid surfaces, such that the surface can take the place of one of the remaining coordination sites $[9,10,11]$. Usually the energy barrier for magnetization reversal in these molecules is much smaller than the thermal energy, in particular for ambient temperature. The time- or ensemble-averaged magnetization of such paramagnetic molecules is thus zero without external field. It has been realized, though, that when placed on a ferromagnetic substrate, even at room temperature a sizeable magnetization can be detected. Element-resolved x-ray magnetic circular dichroism (XMCD) experiments on Mn tetraphenyl-porphyrin (MnTPP) on Co films [12] and on Fe octaethyl porphyrin (FeOEP, see inset of Fig. 1) on Co and Ni films [13] showed a sizeable difference in the absorption of right and left circularly polarized soft $\mathrm{x}$ rays at the absorption edge of the central ion species even at room temperature and without any external magnetic field. This XMCD difference provides information about the magnetic properties of the corresponding element in the sample [14], and can be 
conveniently used to measure magnetic properties of a submonolayer of adsorbates on top of a magnetic substrate, provided adsorbate and substrate contain different elements. The presence of XMCD at the absorption edge of the molecule's ion in zero external magnetic field can only be explained by a magnetic coupling between the remanently magnetized substrate and the magnetic moments in the adsorbed metalloporphyrin molecules. The dipolar coupling from the substrate, which is present close to the surface due to the discrete positions of the atomic magnetic moments in the metal layer, is on the average typically only of the order of some $\mathrm{mT}$, as estimated in the supplementary material of [15], and thus much smaller than the experimentally observed coupling presented in the following.

Spectra of 0.6 atomic monolayers (ML) of FeOEP on $\mathrm{Ni} / \mathrm{Cu}(001)$ are shown in Fig. 1 as black broken lines. On the left, spectra taken at the $\mathrm{Fe} L_{2,3}$ edges are shown, on the right the corresponding spectra at the $\mathrm{Ni} L_{2,3}$ edges. The top row displays the absorption spectra, averaged for positive and negative helicity, while the bottom row presents the XMCD difference spectra (absorption at positive helicity minus absorption at negative helicity). The nonvanishing XMCD in the Fe $L_{2,3}$ spectra proves the magnetic substrate coupling, while the identical sign of the XMCD at the Fe and $\mathrm{Ni} L_{2,3}$ edges shows that molecule and substrate magnetization are aligned parallel.

Density functional theory (DFT) calculations for Fe porphyrin (FeP) on Co revealed that this coupling is mediated by a $90^{\circ}$ superexchange via the nitrogen atoms. This is schematically shown in Fig. 2 (a), which reproduces the result of a DFT calculation for an $\mathrm{FeP}$ molecule adsorbed on $\mathrm{Co} / \mathrm{Cu}(001)$ [13]. Blue and orange colors represent spin density contour surfaces of opposite sign, while red, yellow, and green spheres mark the position of nitrogen, carbon, and hydrogen atoms, respectively, in the molecule. While there is no overlap between the spin densities of equal sign of the Fe ion in the molecule and the Co atoms in the substrate, the indirect $90^{\circ}$ coupling via the spin density of the $\mathrm{N}$ atoms of opposite sign is clearly visualized.

This coupling seems to be rather common for planar transition metal porphyrin and phthalocyanine molecules adsorbed on metallic ferromagnets and has been observed also in other combinations of transition metal ion and substrate $[17,18,19,20]$. In the case of the larger $4 f$ ions, which due to their ionic radius do not fit into the plane of the molecules, double-decker variants exist. In phthalocyanine double deckers one rare-earth ion is coordinated by two parallel phthalocyanine $(\mathrm{Pc})$ units. $\mathrm{TbPc}_{2}$ double-decker molecules show single-molecule-magnet behavior [21, 22]. Also for these molecules a magnetic coupling to a ferromagnetic Ni film [15], to a Co film [23], or to an Fe film as a substrate has been reported [24], in each case with an opposite sign compared to the case of planar transition-metal molecules. The opposite sign of the coupling, antiferromagnetic instead of ferromagnetic, has been attributed to the larger separation of the magnetic ion from the surface, which changes the superexchange coupling path more towards $180^{\circ}$ compared to the planarly coordinated transition metal ions [15]. This coupling has been also studied for other lanthanide ions in double [25] and even triple deckers [26]. Antiferromagnetic coupling is also observed for Cr porphyrin molecules 


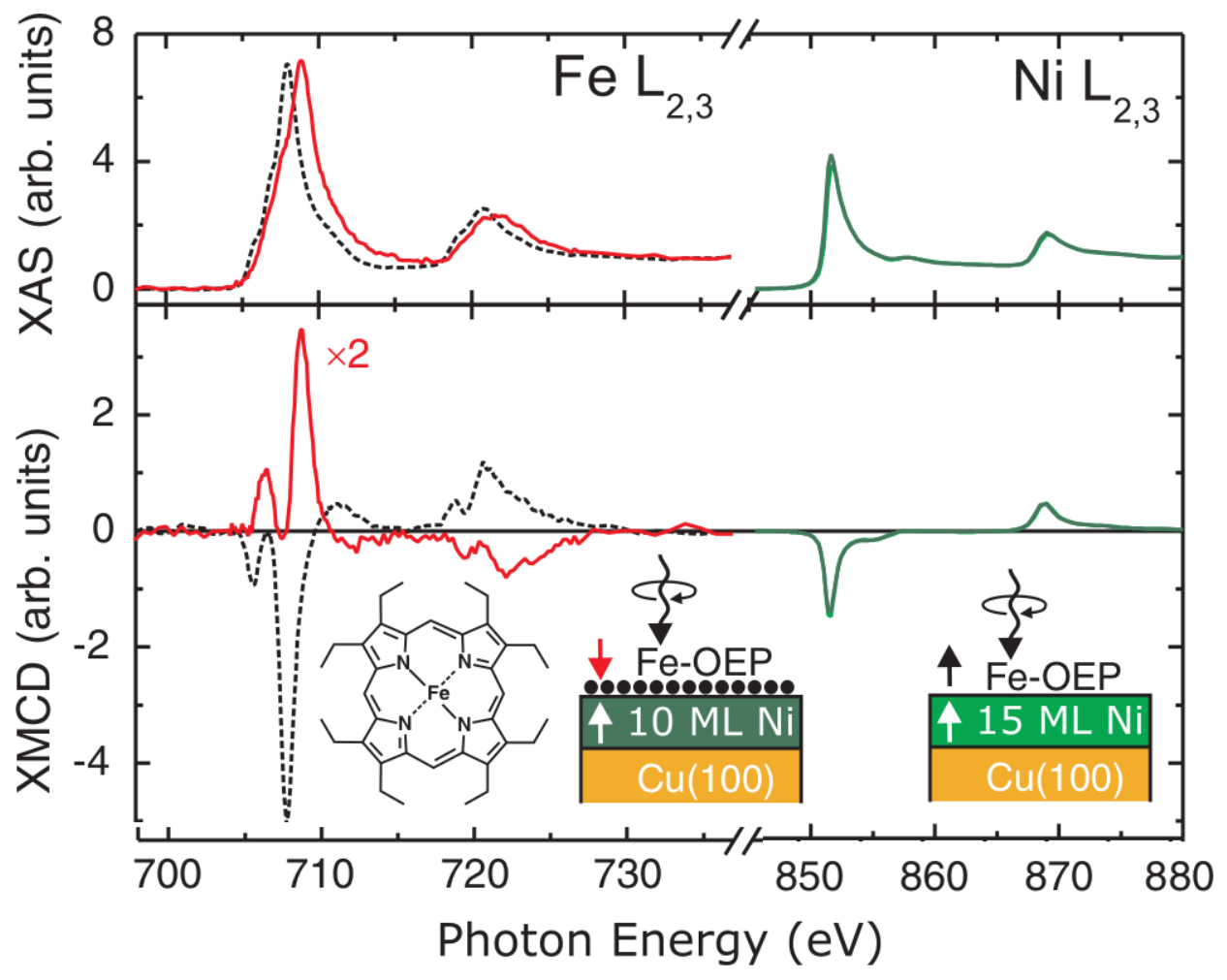

Figure 1. X-ray absorption spectra at the Fe (left) and Ni $L_{2,3}$ edges (right) and the corresponding XMCD difference curves (bottom) of 0.6 ML FeOEP on Ni (black broken lines) and on $\mathrm{O}-\mathrm{Ni}$ (red continuous lines) taken at a sample temperature of 40 $\mathrm{K}$ without external magnetic field. The XMCD changes sign when oxygen atoms are placed between the ferromagnetic Ni substrate and the FeOEP molecules. The inset shows sketches of the Fe porphyrin molecule and of the samples. After [16]. Copyright 2009 by the American Physical Society.

on a Co substrate, where the antiparallel exchange coupling is attributed to the less than half-filled $3 d$ shell of the $\mathrm{Cr}^{2+}$ ion [27], and for $\mathrm{Mn}$ phthalocyanine molecules on ferromagnetic EuO, where the interaction between the half-filled $3 d$ shell of $\mathrm{Mn}^{2+}$ and the $\mathrm{Eu} 5 d$ electrons is held responsible for the antiparallel coupling [28]. The coupling of a Cu-tetraazaporphyrin to a magnetite(100) surface changes even sign as a function of the magnetization direction, which is interpreted as evidence for a strongly anisotropic exchange coupling between the $\mathrm{Cu}$ moment and the magnetite surface resulting from the simultaneous presence of competing superexchange coupling paths [29].

For late-transition-metal porphyrins the coupling to the substrate is ferromagnetic as long as these molecules are placed directly on top of a metallic $3 d$ ferromagnet. This is no longer true when other atoms are inserted between substrate and molecules. If oxygen atoms, for example, are adsorbed on top of the magnetic substrate, the sign of the coupling reverses. This is shown in Fig. 1 by the spectra reproduced by red and green lines. The spectra of the Ni substrate are identical to the case with no oxygen on the sample, but the XMCD at the Fe absorption edges (left bottom) reverses sign. A surfactant effect described in literature helps to prepare a regular array of oxygen atoms 


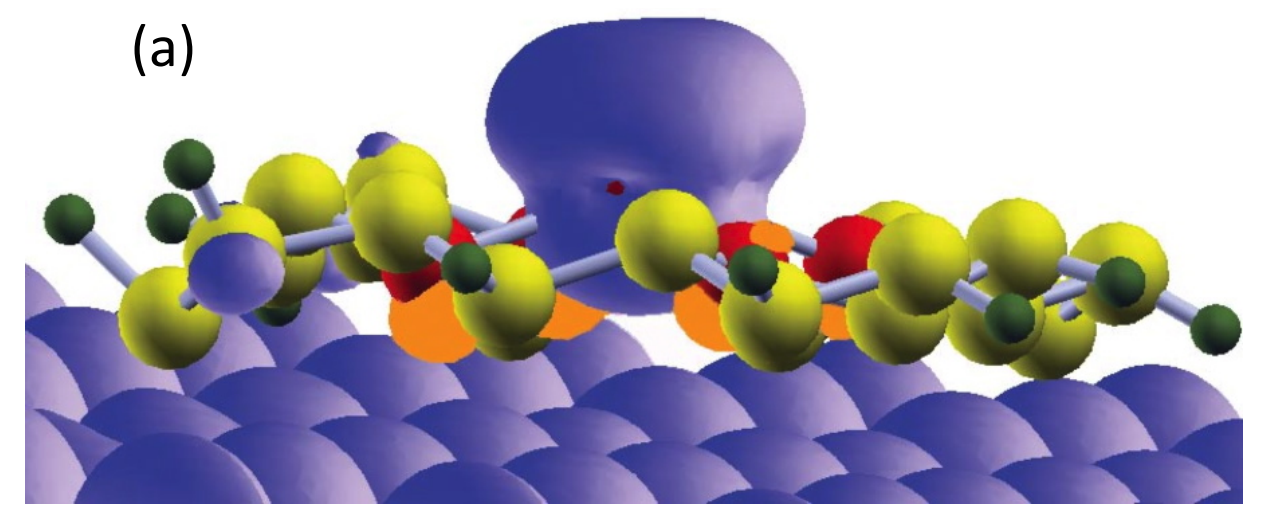

(b)

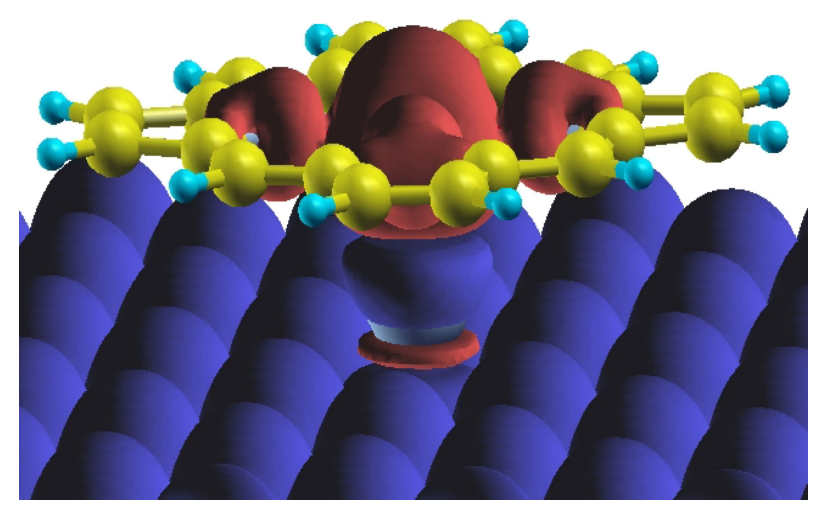

Figure 2. (a) Calculated spin density contours of an FeP molecule adsorbed on a $\mathrm{Co}(001)$ substrate. Blue and orange are spin density contour surfaces of opposite sign, while red, yellow, and green spheres mark the position of nitrogen, carbon, and hydrogen atoms, respectively. Reprinted from [13] by permission from Macmillan Publishers Ltd: Nature Materials, copyright 2007. (b) Calculated spin density contours of an $\mathrm{FeP}$ molecule adsorbed on top of an oxygen molecule on a $\mathrm{Co}(001)$ substrate. Blue and red are spin density contour surfaces of opposite sign, while yellow and cyano spheres mark the position of carbon and hydrogen atoms, respectively. Reprinted figure with permission from [16]. Copyright 2009 by the American Physical Society. In both cases the $\mathrm{Fe}$ ion is coupled by superexchange coupling to the ferromagnetic Co substrate. In (a) a $90^{\circ}$ superexchange coupling via the nitrogen atoms is leading to a ferromagnetic coupling between molecule and substrate, while in (b) a $180^{\circ}$ superexchange coupling via the underlying oxygen atom results in an antiferromagnetic coupling.

on top of $\mathrm{Co}$ or $\mathrm{Ni}$ films on $\mathrm{Cu}(001)$ [30,31]: If the clean $\mathrm{Cu}(001)$ surface is exposed to oxygen at a certain elevated temperature, the adsorbed oxygen atoms float at the surface during subsequent room-temperature deposition of $\mathrm{Co}$ or $\mathrm{Ni}$, forming a regular $(2 \times 2)$ superstructure.

DFT calculations have helped to understand this sign reversal by the interleaved oxygen atoms: While in the case with no oxygen atoms the coupling is identified as $90^{\circ}$ superexchange coupling, in the case of a metal porphyrin molecule sitting on top of an adsorbed oxygen atom, the coupling mechanism is a $180^{\circ}$ superexchange coupling 
[16], similar to the one that leads to antiferromagnetism in $3 d$ monoxides like $\mathrm{CoO}$ or $\mathrm{NiO}$. This is illustrated in Fig. 2 (b), which shows the opposite signs of the calculated spin density in red and blue. The spin density of the metal ion in the molecule at the top is connected along a straight vertical path down to the substrate's spin density in a sign-alternating way (red-blue-red-blue). This is the typical appearance of a $180^{\circ}$ superexchange coupling.

The coupling mechanism becomes even more complex if a layer of graphene [32] is inserted between metalloporphyrin molecules and a metallic ferromagnet. The $s p_{2^{-}}$ hybridized carbon atoms in graphene are not supposed to engage in covalent bonds with adsorbed molecules, which are considered to be purely physisorbed on graphene. DFT calculations considering van-der-Waals corrections consequently yield a relatively large distance of $3.51 \AA$ between the Co ion in the molecule and the graphene carbon atoms [33]. Co $L_{2,3}$ absorption spectra are nearly identical to the ones of CoOEP bulk material, and significantly different to the ones of CoOEP adsorbed on $\mathrm{Ni}$ ([33], supporting information), confirming a negligible influence of the adsorption to the graphene substrate on the Co electronic states. Nevertheless, experiments show that CoOEP molecules on graphene couple magnetically to the Ni film on which the graphene is grown [33]. The graphene layer has been prepared on a $\mathrm{Ni}$ film deposited on a $\mathrm{W}(110)$ single crystal surface, following a recipe from literature [34, 35]. Again, a nonvanishing XMCD signal at the Co $L_{2,3}$ absorption edges shows that even in this system an antiferromagnetic coupling between the Ni layer and the Co ions is present. Fig. 3 presents the theoretically calculated spin and charge densities of a CoP molecule adsorbed to graphene/ $\mathrm{Ni}$. Looking only at the spin density (top panel), one could be tempted to interpret the coupling as direct overlap of the spin density at the site of the Co ion, with a shape indicating a predominant $d_{3 z^{2}-r^{2}}$ orbital character, and the induced spin density in the graphene layer of the same sign (blue contours). However, considering also the charge density distribution (bottom panel), one realizes that the coupling path is more complicated: The small spin density on graphene, antiparallel to the dominant one on $\mathrm{Ni}$, is induced by hybridization of spin-minority $\mathrm{Ni} s p$ states with graphene $p_{z}$ orbitals. A weak antiparallel coupling between graphene and porphyrin $\pi$ orbitals then induces a small spin density in the molecule, mainly on the pyrolic nitrogen atoms, parallel to the Ni magnetization, recognized in Fig. 3 (a) by yellow contours. The final chain in the exchange path is the magnetic coupling between the nitrogen atoms and the central Co ion, mediated by a weak hybridization with the Co $d_{3 z^{2}-r^{2}}$ orbital, favoring an antiparallel spin polarization on $\mathrm{N}$ and Co.

An estimate of the coupling strength can be obtained from the temperature dependence of the XMCD signal, measured in zero field at remanence of the magnetic substrate. The temperature dependence of the substrate magnetization is taken into account by normalizing the molecule's XMCD signal to it, thereby utilizing the fact that the time constant for exchange coupling is significantly smaller than the time constant of typical thermal fluctuations of the substrate magnetization. Since the magnetic anisotropy of magnetic molecules coupled to a ferromagnetic substrate is not easy to 


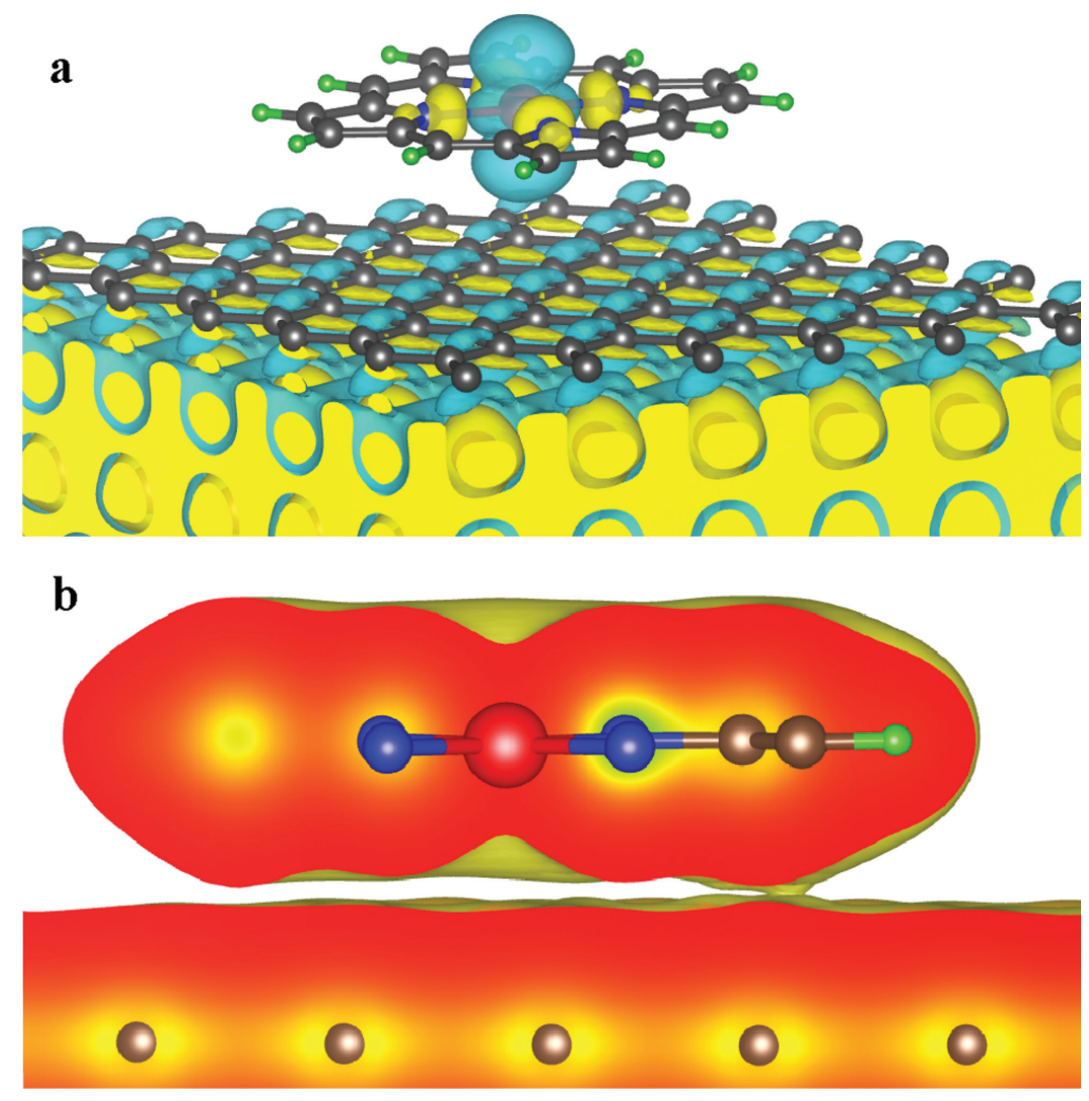

Figure 3. (a) Calculated spin density contours of a $\mathrm{CoP}$ molecule adsorbed on a graphene layer grown on $\mathrm{Ni} / \mathrm{W}(110)$. Blue and yellow mark spin density contour surfaces of opposite sign, while grey and green spheres mark the position of carbon and hydrogen atoms, respectively. (b) Cut through the calculated charge density contour of the same system. Red, blue, golden, and green spheres represent Co, N, C, and $\mathrm{H}$ atoms, respectively. Overlapping charge densities between the CoP molecule and the graphene layer are seen mainly at the outer ligands of the molecule. From [33], copyright 2013 Wiley-VCH Verlag GmbH \& Co.

measure, in experimental estimates of the coupling energy based on the temperature dependence it is mostly assumed to be zero. The temperature dependence of the XMCD is then fitted by a Brillouin function, assuming the behavior of an isotropic quantummechanic magnetic moment. Coupling energies defined as half of the energy difference between parallel and antiparallel coupling obtained in this way are $70 \mathrm{meV}$ for FeOEP on $\mathrm{Co}, 20 \mathrm{meV}$ on $\mathrm{Ni}$ [36], and $37 \mathrm{meV}$ for FeOEP on oxygen-covered Co [16]. The coupling energy of CoOEP to $\mathrm{Ni}$ across graphene is smaller, but still amounts to 1.8 $\mathrm{meV}[33]$.

A graphene layer can thus obviously be used to mainly decouple magnetic molecules from a reactive metallic ferromagnetic surface, while still allowing for magnetic interaction. The latter could be used to control the direction of the molecule's magnetic moment. The cage of carbon Buckminster fullerenes $[37,38]$ can be regarded as a spherical edition of graphene. Magnetic coupling across the fullerene cage, similar to 


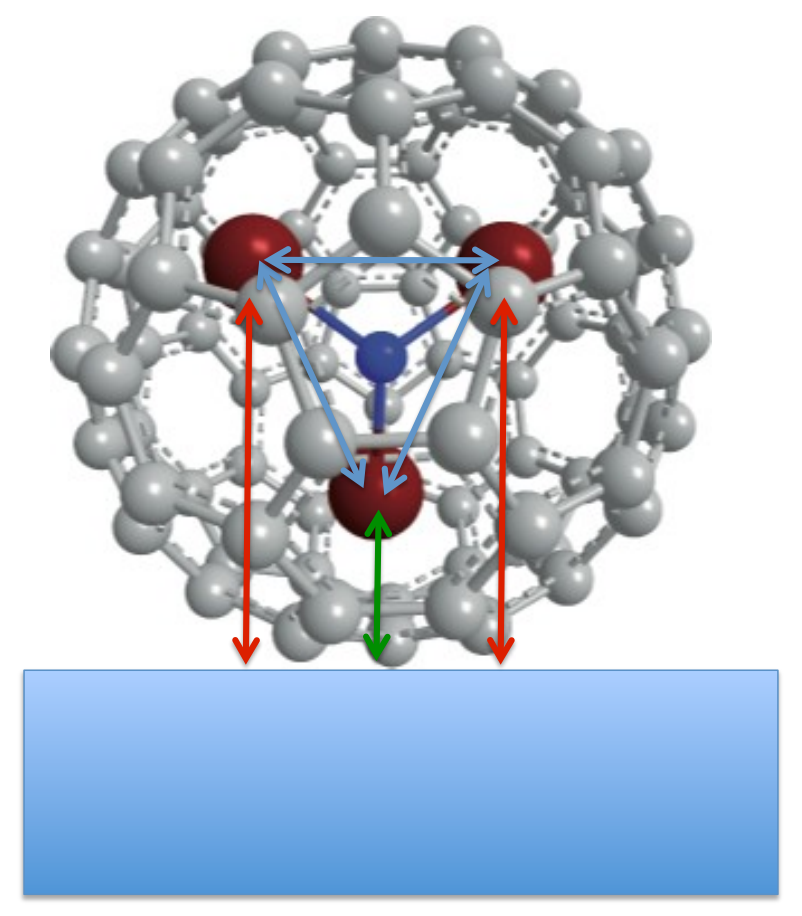

Figure 4. Sketch of a $\mathrm{Gd}_{3} \mathrm{~N} @ \mathrm{C}_{80}$ molecule adsorbed on a magnetic substrate. Red, blue, and grey spheres represent Gd, N, and C atoms, respectively. The superimposed arrows indicate magnetic coupling between the Gd atoms as well as to the substrate.

the one across a graphene layer, is thus conceivable. The inner free space of fullerenes offers the opportunity to encapsulate magnetic atoms or ions, for example to protect them from a reactive environment. Fullerenes filled with atoms or clusters are called "endohedral fullerenes" [39]. In the case of trimetallic nitride endohedral fullerenes, three rare-earth ions are contained in a relatively small space. Long magnetic relaxation times, characteristic of single-molecule magnets, have been observed in $\mathrm{DySc}_{2} \mathrm{~N} @ \mathrm{C}_{80}$ endohedral fullerenes [40]. Magnetic coupling to a substrate has been studied in $\mathrm{Gd}_{3} \mathrm{~N} @ \mathrm{C}_{80}$ adsorbed on $\mathrm{Ni} / \mathrm{Cu}(001)$ [41]. A sketch of this molecule is shown in Fig. 4. The observed complicated behavior of the XMCD signal at the $\mathrm{Gd} M_{5}$ edge as a function of external magnetic field and temperature revealed that in addition to a parallel intramolecular magnetic coupling between the three $\mathrm{Gd}$ ions inside the molecule (blue arrows in Fig. 4), there are also (at least) two different kinds of magnetic interactions active that couple the Gd moments to the substrate ones. The experimental data can be explained by the presence of a stronger ferromagnetic coupling acting on a smaller part of the Gd moments, and a weaker antiferromagnetic one, acting on a larger part of the Gd moments. Two different scenarios are equally consistent with the data [41]: In the first, $43 \%$ of the Gd moments couple ferromagnetically to the Ni substrate with a coupling energy of $6.1 \mathrm{meV}$, and $57 \%$ couple antiferromagnetically with a coupling energy of $2.2 \mathrm{meV}$, while $\mathrm{Gd}$ moments inside the molecule are coupled together ferromagnetically with an energy of $50 \mu \mathrm{eV}$. In this case, the different fullerene species would correspond to molecules with different adsorption orientations. In the second scenario, two out 
of the three $\mathrm{Gd}$ ions in each molecule couple antiferromagnetically to the substrate, while the third one couples ferromagnetically. Coupling energies in this case would be 5.1 and $2.0 \mathrm{meV}$ for the two species, respectively, while the intramolecular coupling amounts to $137 \mu \mathrm{eV}$. This second scenario is depicted by arrows in Fig. 4, where red arrows indicate a weaker antiferromagnetic coupling, green stands for a stronger ferromagnetic coupling, and blue for the intramolecular ferromagnetic coupling between $\mathrm{Gd}$ ions. The coupling energies across the fullerene cage of a few meV are of the same order of magnitude as for the coupling of metal porphyrin molecules across graphene. The coupling mechanism is likely similar to the one across graphene, namely an indirect carbon-cage-mediated exchange. Such a magnetic substrate coupling should not only be specific to the case of $\mathrm{Gd}_{3} \mathrm{~N} @ \mathrm{C}_{80}$, but more generally valid likewise also for other, similar endohedral fullerenes, allowing a reliable communication with and access to the enclosed magnetic units of endohedral fullerenes without the need for an applied magnetic field.

Coupling between planar magnetic molecules and ferromagnetic films is thus possible across a variety of interlayers. Even across a nonmagnetic metallic spacer layer such as $\mathrm{Cu}$, clear indications for coupling to adsorbed $\mathrm{Mn}$ phthtalocyanine molecules have been observed [42]. Although there is no more direct contact between the molecules and the ferromagnetic layer, the electronic states close to the Fermi edge in the nonmagnetic overlayer acquire a spin polarization, the sign of which oscillates as a function of the overlayer thickness and couple to the magnetic moment of the molecules. This can be viewed in terms of the spin polarization of quantum well states in the nonmagnetic layer emerging due to the different confinement of electrons that are of the majority or minority type in the adjacent ferromagnetic layer [43, 44, 45]. This interlayer exchange coupling [46, 47] is well explored both experimentally [48, 49, 50] and theoretically $[51,52,53]$ in trilayered systems in which the magnetizations of two ferromagnetic layers couple across a nonmagnetic spacer layer. In the case of molecules adsorbed on top of the nonmagnetic layer, the coupling path to the metal substrate connects to the electronic states of the nonmagnetic thin film, which feel the spin polarization of the buried ferromagnetic layer in an oscillatory dependence on the thickness of the nonmagnetic layer.

Apart from coupling to ferromagnetic substrates, also coupling between individual molecules and antiferromagnetic substrates is possible $[24,54]$. In antiferromagnetic materials the direction of neighboring atomic moments changes such as to yield zero total magnetization. If adsorbed molecules were to randomly couple to the moments of a magnetically compensated surface, an ensemble-averaging method such as XMCD could not detect the coupling. However, if the system is cooled in a magnetic field through the ordering temperature of the antiferromagnet, the Néel temperature, and there is coupling between ferromagnetic moments and some of the surface magnetic moments in the antiferromagnet, a certain domain structure will be imposed in the antiferromagnet upon cooling such as to favor the alignment of the ferromagnetic moments along the cooling field direction. This results in a unidirectional shift of the magnetization curves along the field axis. For bilayers of a ferromagnetic and an antiferromagnetic 
material this is well-known as the "exchange bias" effect $[55,56]$. XMCD field curves of $\mathrm{TbPc}_{2}$ on antiferromagnetic $\mathrm{Mn}$ films on $\mathrm{Ag}(001)$ [54] as well as on FeMn films on $\mathrm{Cu}(001)$ [24] showed a small loop shift, which proves that some coupling to the antiferromagnetic surface must be present. Stabilizing the spin of adsorbed molecules by an antiferromagnetic substrate rather than a ferromagnetic one may have the advantage of greater insensitivity to external magnetic fields.

Recently even the coupling of nonplanar magnetic molecules such as the spincrossover molecules discussed in Sec. 4 to a ferromagnetic Co film has been observed [57]. Since the Fe ion in this molecule is separated from the Co surface by as much as $5.1 \AA$, also here the coupling has to be of the superexchange type, mediated by the ligands touching down to the substrate surface.

\section{Controlling the molecular magnetic properties by on-surface chemical modification}

When a planar, four-fold-coordinated metal complex like a metalloporphyrin or phthalocyanine is adsorbed on a surface in a parallel, flat way, there is free access to the metal ion from the top side, opposite to the surface. This remaining sixth coordination site can be used to gain control on the magnetic properties of the molecule. By attaching a small molecule as additional ligand to this site, the crystal field felt by the metal ion is modified, and thus also the electronic and magnetic properties of the molecule including the coupling to the substrate. X-ray and UV photoelectron spectroscopy measurements have shown that the adsorption of NO on top of Co tetraphenylporphyrin (TPP) molecules on $\mathrm{Ag}(111)$ in ultra-high vacuum weakens the binding between Co and the Ag substrate [58]. In this study, $300 \mathrm{~L}$ of NO gas was dosed to the sample held at a temperature of $140 \mathrm{~K}\left(1 \mathrm{~L}=10^{-6}\right.$ mbar $\left.\mathrm{s}\right)$. This was interpreted as a competition between the NO molecule and the Ag surface as two axial ligands of the Co ion, similar to the trans effect [58]. After thermal desorption of the $\mathrm{NO}$ at $500 \mathrm{~K}$, the previous situation is recovered, demonstrating a reversible manipulation of the Co electronic properties by this chemical stimulus.

When the same molecule is adsorbed on a ferromagnetic Ni film, the magnetic substrate coupling discussed in the previous section leads to a nonvanishing magnetization of the adsorbed molecules and thus XMCD signal at the Co $L_{2,3}$ absorption edges even at room temperature. After dosing $6000 \mathrm{~L}$ of NO to $1 \mathrm{ML}$ of CoTPP on $\mathrm{Ni} / \mathrm{Cu}(001)$ at room temperature, this XMCD signal entirely disappears [18]. This is a clear indication that the magnetism of adsorbed metalorganic molecules can be strongly influenced by chemically disturbing the metal ion's electronic system, for example by coadsorption of small molecules like nitric oxide. After heating the sample to $615 \mathrm{~K}$, an XMCD signal reappeared, indicating the removal of NO from the site of the Co ion [18].

The presence of NO molecules even after heating of the sample at regions where the bare metallic substrate is exposed can be avoided if these regions are covered 

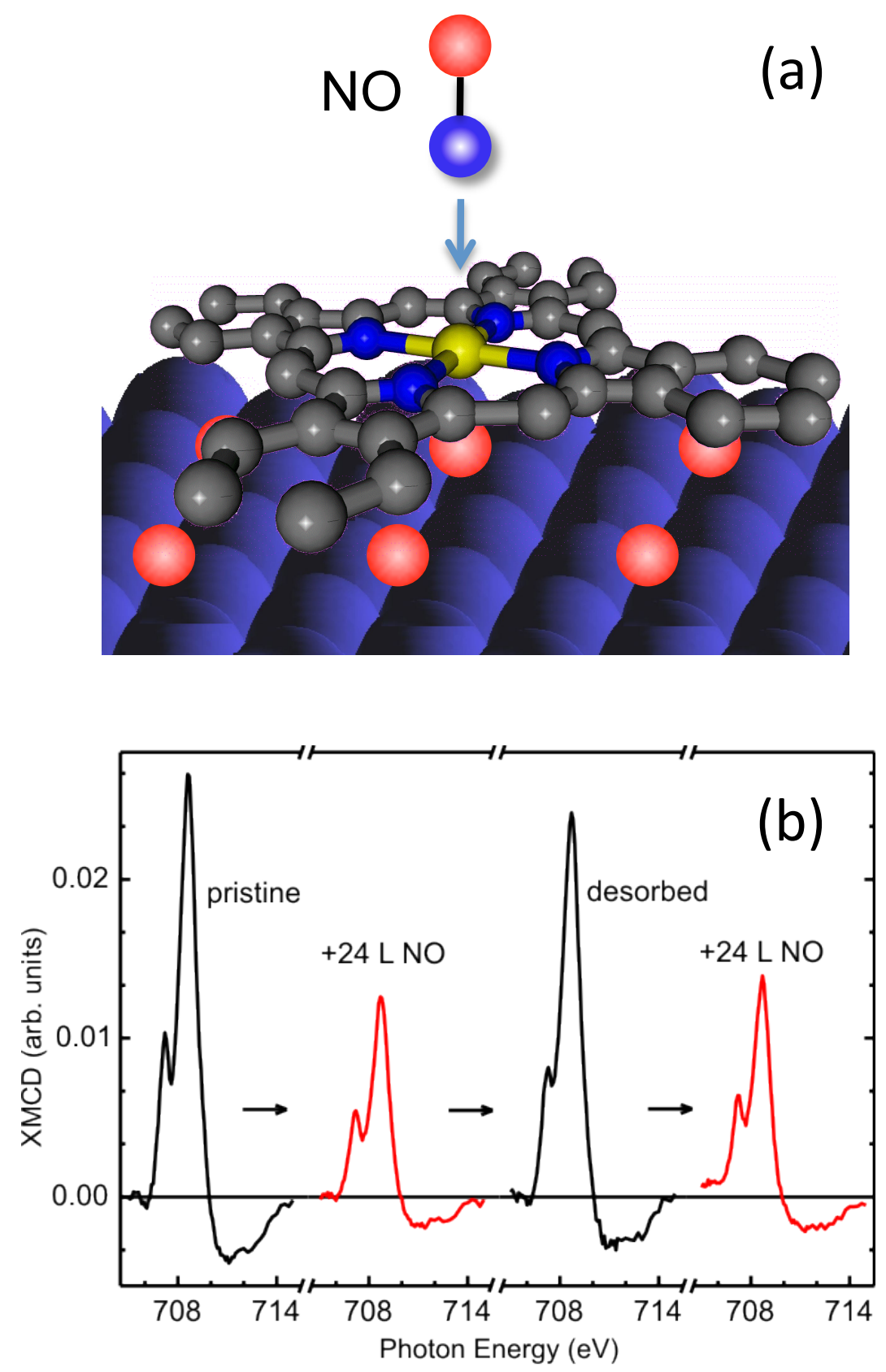

Figure 5. (a) Sketch of an NO molecule adsorbing on an FeOEP molecule adsorbed on a $(2 \times 2)$-O-covered $\mathrm{Co}(001)$ film. Hydrogen atoms of the FeOEP molecule are not shown for clarity. (b) Sequence of XMCD difference spectra at the Fe $L_{3}$ edge of 0.6 ML FeOEP on $\mathrm{O} / \mathrm{Co} / \mathrm{Cu}(001)$ measured in the pristine state, after dosage of $24 \mathrm{~L}$ of $\mathrm{NO}$, after thermal desorption of the $\mathrm{NO}$ at $350 \mathrm{~K}$, and after dosing with $24 \mathrm{~L}$ of NO once more (from left to right). Sample temperature during the measurements was 120 K. Reprinted with permission from [59]. Copyright 2011 American Chemical Society. 
by an ordered array of oxygen atoms. On $\mathrm{Ni} / \mathrm{Cu}(001)$ or $\mathrm{Co} / \mathrm{Cu}(001)$, this can be achieved by depositing the $\mathrm{Ni}$ or Co films, respectively, on a preoxidized $\mathrm{Cu}(100)$ single crystal, following Ref. [31]. The oxygen atoms act as a surfactant for the growth of the ferromagnetic film, always floating on top of the surface [31, 30]. This results in a wellcharacterized $(2 \times 2)$ superstructure of $0.5 \mathrm{ML}$ atomic oxygen on top of the ferromagnetic films. As discussed in the previous section, the magnetic coupling of adsorbed porphyrin molecules is present also through such a layer of oxygen atoms [16]. Fig. 5 shows the XMCD signal at the Fe $L_{3}$ absorption edge of 0.6 ML of FeOEP molecules adsorbed on $c(2 \times 2)-\mathrm{O} / \mathrm{Co} / \mathrm{Cu}(001)$ at several stages of sample history. From left to right, the spectra correspond to the pristine sample, to the sample after adsorbing $24 \mathrm{~L}$ of $\mathrm{NO}$ at $120 \mathrm{~K}$, after desorbing the $\mathrm{NO}$ at $350 \mathrm{~K}$, and after anew adsorbing $24 \mathrm{~L} \mathrm{NO}$ at $120 \mathrm{~K}$ [59]. All spectra have been taken at $120 \mathrm{~K}$. It is evident that the adsorption of NO causes the reduction of the XMCD signal by about a factor of 2 , that after the desorption of NO from the sample, the initial Fe XMCD intensity is almost completely recovered, and that dosing NO again causes once more a reduction by nearly a factor of 2 . From the fact that the Fe $L_{2,3}$ absorption signal hardly changes after adsorption of NO it had been concluded that the main effect of the NO in this system was the reduction of the magnetic coupling between the Fe ion in the porphyrin molecules and the magnetic Co substrate, which at finite temperatures results in a reduction of the molecule's magnetization [59].

The situation is different in CoOEP molecules on $c(2 \times 2)-\mathrm{O} / \mathrm{Ni} / \mathrm{Cu}(001)$ [60], as illustrated in Fig. 6. It shows absorption spectra at the Co $L_{2,3}$ edges of CoOEP adsorbed on a $\mathrm{Ni}$ ferromagnetic substrate on $\mathrm{Cu}(001)$, covered with a $(2 \times 2)$ oxygen layer. From bottom to top, spectra taken from the pristine sample, after dosing $28 \mathrm{~L}$ of NO at 130 $\mathrm{K}$, after desorbing the NO at $350 \mathrm{~K}$, and after a second dosage of NO are shown [60]. By using linearly $p$-polarized $\mathrm{x}$ rays, this experiment is mostly sensitive to unoccupied outof-plane $d$ orbitals of Co. The spectra exhibit a clear shift in energy, which is completely reversible upon removal of the NO by thermal desorption. This shift is explained by charge transfer from the Co $d_{z^{2}}$ orbital to NO, leading to a further partial oxidation of the $\mathrm{Co}^{2+}$ ion [60]. The consequence is a reduction of the magnetic moment of the Co ion, which in the $d^{7}$ low-spin state is due to the unpaired electron in the $d_{z^{2}}$ state.

Comparing the two systems FeOEP/O-Co and CoOEP/O-Ni, the effect of NO adsorption to the porphyrin molecules in the former is mainly the reduction of the magnetic coupling between the molecule and the ferromagnetic substrate, while in the latter there is also a prominent charge transfer away from the metal ion. Both leads to an about $50 \%$ reduction of the magnetization of the molecules at the measurement temperature of $130 \mathrm{~K}$. The different behavior may be explained by the different oxidation and spin states in the two systems: The Fe ion in FeOEP/O-Co is in a $3+$ oxidation state and intermediate spin state, where an oxygen atom of the substrate takes the role of the fifth ligand [59], such that further oxidation is unlikely and the NO is only physisorbed. In $\mathrm{CoOEP} / \mathrm{O}-\mathrm{Ni}$, in contrast, $\mathrm{Co}$ is in a $2+$ oxidation state and low spin state, and may more easily experience further oxidation by the attached NO. 


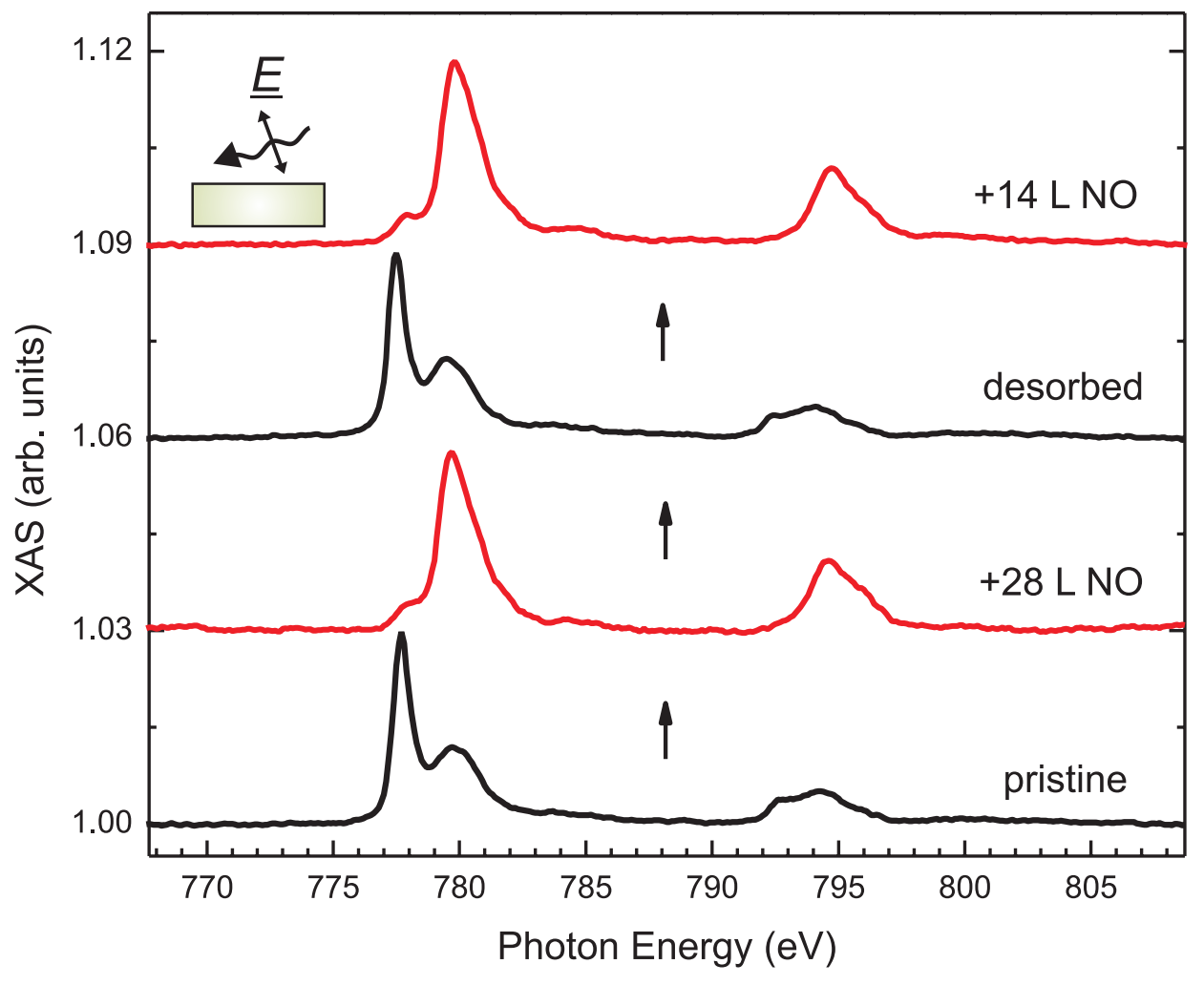

Figure 6. Co $L_{2,3}$ absorption spectra of $0.7 \mathrm{ML} \mathrm{CoOEP}$ on $(2 \times 2)-\mathrm{O} / \mathrm{Ni} / \mathrm{Cu}(001)$ measured with linearly $p$ polarized light at an angle of $20^{\circ}$ between the incoming $\mathrm{x}$ rays and the surface at $130 \mathrm{~K}$ for the pristine sample, after dosing with $28 \mathrm{~L}$ of NO, after the ensuing desorption of $\mathrm{NO}$ by heating to $350 \mathrm{~K}$, and after dosing again with $14 \mathrm{~L}$ of NO (from bottom to top). Measurement temperature $130 \mathrm{~K}$. The spectra are shifted vertically for clarity. The sketch in the inset illustrates the measurement geometry and the orientation of the polarization vector $E$ of the x rays. From [60].

Both, a spin state change and a change in the interaction with the underlying substrate, has been reported for $\mathrm{Mn}$ phthalocyanine $(\mathrm{MnPc})$ molecules on a $\mathrm{Bi}(110)$ surface to which CO is adsorbed [61]. Scanning tunneling spectroscopy revealed a variation of the Kondo screening of the magnetic moment in the Bi substrate, which is an indication for the variation of the substrate-molecule interaction. Accompanying first principles calculations showed that also the Mn spin state is changed from $S=1$ to $S=1 / 2$ upon CO attachment [61]. All these changes could be reversed upon desorption of the $\mathrm{CO}$ molecules.

Depending on the metal center of the magnetic molecule and the adsorbing small molecule quite different effects on the molecular magnetization can be attained. Besides a full or partial quenching of the magnetization of metalloporphyrin molecules coupled to a ferromagnetic substrate, also its reversal or enhancement is possible. In Co tetraphenylporphyrin (CoTPP) molecules on $\mathrm{Ni} / \mathrm{Cu}(001)$, adsorption of $\mathrm{NO}$ leads to the disappearance of the molecular magnetization, while in $\mathrm{FeTPP} / \mathrm{Ni} / \mathrm{Cu}(001)$ it is only partly quenched [62]. Interestingly, in $\mathrm{MnTPP} / \mathrm{Co} / \mathrm{Cu}(001)$, the $\mathrm{Mn}$ magnetization after 
NO adsorption is reduced, but also reversed in sign with respect to the Co substrate magnetization [62]. Furthermore, adsorption of ammonia to Mn phthalocyanine on $\mathrm{Co} / \mathrm{Cu}(001)$ leads to an enhancement of the Mn magnetization at low temperatures, but a decrease of the coupling strength to the substrate [62]. Theoretical density functional calculations could reproduce these different effects of adsorbing small molecules to surface-coupled square-planar metal complexes. They show that for different metal centers the bond angle of $\mathrm{NO}$ as well as the variation of the distance between the metal center and the substrate depend on the $3 d$ electronic configuration, thus leading to the observed variety of effects [62].

Instead of switching off the spin of an adsorbed metal porphyrin molecule by coadsorption of a small molecule, also the opposite effect has been observed and is possible. Adsorbed NiTPP molecules are in an $S=0$ low-spin state on $\mathrm{Co} / \mathrm{Cu}(001)$. Coadsorption of $\mathrm{NH}_{3}$ leads to the emergence of a sizable XMCD peak at the $\mathrm{Ni} L_{2,3}$ edges [63]. The ammonia can be thermally desorbed, such that this switching on of the Ni spin is fully reversible. Theoretical DFT calculations revealed that the ammonia increases the energy of the previously doubly occupied $\mathrm{Ni} d_{z^{2}}$ orbital, moving it closer to the previously unoccupied $d_{x^{2}-y^{2}}$ orbital, such that after ammonia attachment both these orbitals are singly occupied by an unpaired electron, leading to $S=1$ [63].

A regular two-dimensional assembly of two different species of paramagnetic molecules that are differently susceptible to chemical switching by ammonia coadsorption has been reported in Ref. [64]. Iron phthalocyanine molecules functionalized with 16 fluorine atoms at the outside (perfluorinated iron(II)phthalocyanine, $\mathrm{FeF}_{16} \mathrm{Pc}$ ) engages in hydrogen bonds with a "normal" phthalocyanine molecule, for example MnPc. Coevaporation of both molecules in a 1:1 stoichiometric ratio on a $(2 \times 2)-\mathrm{O} / \mathrm{Co} / \mathrm{Cu}(001)$ substrate leads to a regular chessboard-like arrangement, as shown in Fig. 7 [64]. Both molecules are subject to an antiparallel magnetic coupling to the magnetization of the Co substrate, as evidenced from XMCD measurements taken at $70 \mathrm{~K}$ with zero external magnetic field (Fig. 7). Coadsorption of ammonia leads to the quenching of the moment of the $\mathrm{FeF}_{16} \mathrm{Pc}$ molecules, while the spin of the MnPc molecules is just reduced, but not quenched [64]. After adsorption of $\mathrm{NH}_{3}$, the pattern of magnetic moments on the surface is thus modified. The magnetic moment of each other molecule, namely of all the $\mathrm{FeF}_{16} \mathrm{Pc}$ molecules, has been switched off. Adsorption and desorption of $\mathrm{NH}_{3}$ have been found to be fully reversible [64]. In addition, $\mathrm{NH}_{3}$ displays a stronger affinity to $\mathrm{MnPc}$ compared to $\mathrm{FeF}_{16} \mathrm{Pc}$, which could be an additional means of selective manipulation.

\section{Controlling the magnetic properties of adsorbed spin-crossover molecules}

Spin-crossover (SCO) molecules possess two metastable spin states as a result of the competition between the ligand-field splitting and the spin-pairing energy. In their lowspin state, the $d$ electrons pair up occupying the levels lowest in energy, whereas in their high-spin state the spin is maximized and the electrons occupy all $d$ levels, as shown in Fig. 8 for the $6 d$ electrons of the $\mathrm{Fe}^{2+}$ ion. The metastable balance of the two spin states 


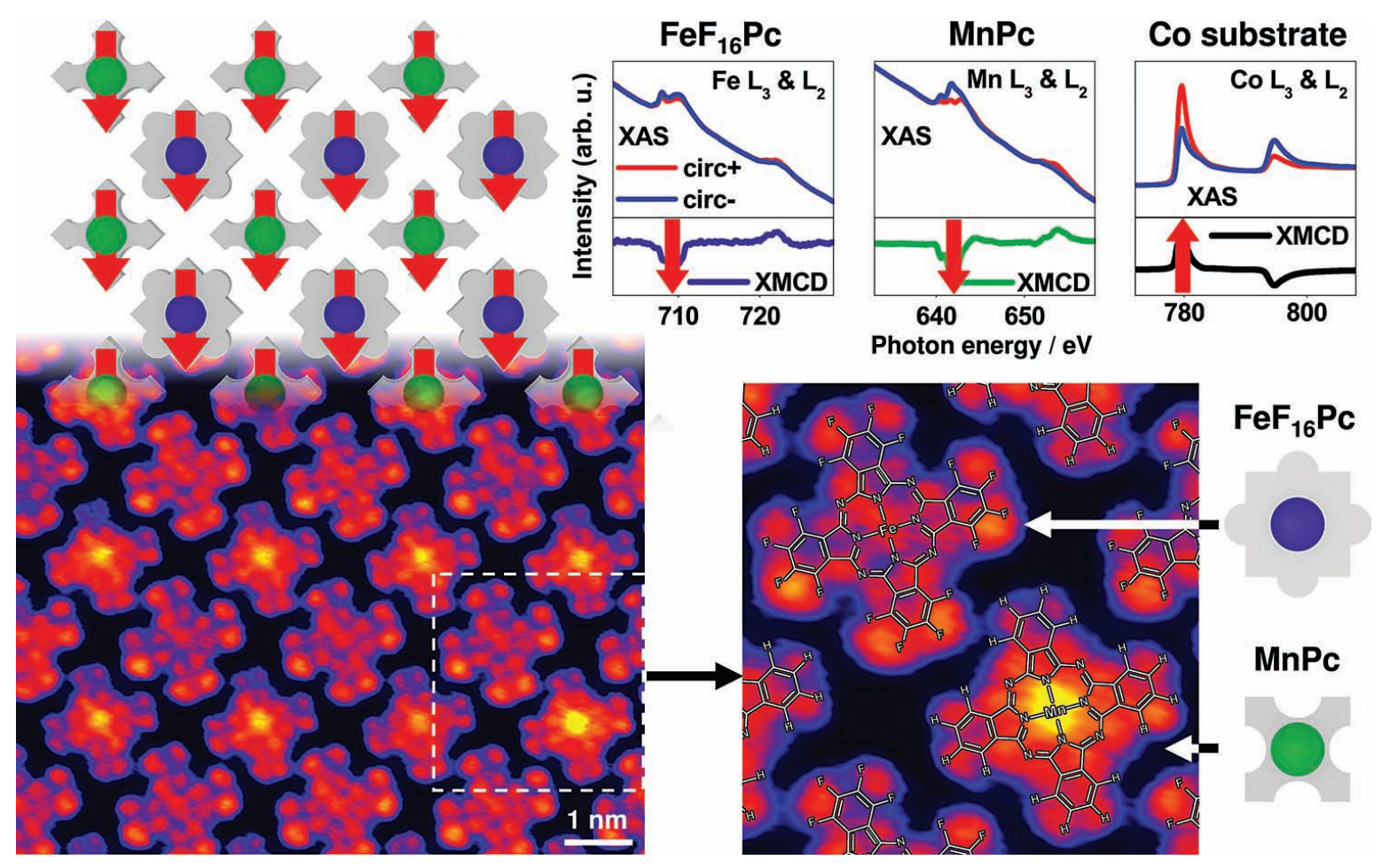

Figure 7. Chessboard-like arrangement of $\mathrm{FeF}_{16} \mathrm{Pc}$ and $\mathrm{MnPc}$ on $(2 \times 2)$ $\mathrm{O} / \mathrm{Co} / \mathrm{Cu}(001)$. Left top: schematic view in which green circles represent $\mathrm{Mn}$, blue circles Fe atoms, and red arrows indicate the direction of magnetic moments coupled antiparallel to the Co substrate magnetization. Bottom: scanning tunneling microscopy image and magnified section thereof with overlaid chemical structure of the molecules. Right top: X-ray absorption spectra and XMCD difference spectra of the Fe, Mn, and Co $L_{2,3}$ edges. Measurement temperature $70 \mathrm{~K}$. From [64], copyright 2013 Wiley-VCH Verlag GmbH \& Co.

\section{Spin Crossover}

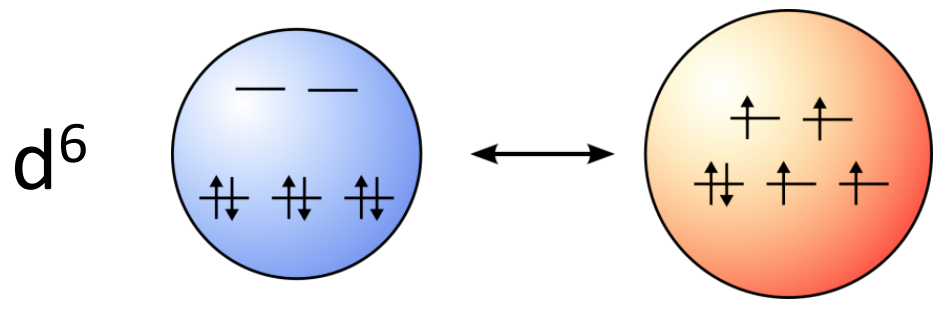

low spin

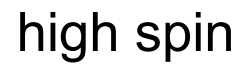

Figure 8. Schematics of the spin-crossover transition in a $3 d^{6}$ system. If the ligandfield splitting of the $d$ states exceeds the spin pairing energy, a low-spin state with $S=0$ is obtained. Otherwise the $d$ states are filled according to Hund's rules, resulting in four unpaired electrons and a high-spin state with $S=2$. Since the entropy is higher in the high-spin state, in spin-crossover compounds it is favored at higher temperatures, whereas the low-spin state is the energetic ground state at low temperatures. 
reacts sensitively to tiny perturbations, such as changes in temperature, intermolecular interactions, or excitation with light.

The temperature-dependent spin transition is driven by the entropy difference between the high-spin and the low-spin state. The entropy difference stems from the difference in spin multiplicity and in the number of accessible vibrational levels. In the high-spin state, the coordination bond is weakened, leading to a closer spacing of the vibrational energy levels and thus to higher entropy. The high-spin state is therefore favored at higher temperatures, while the low-spin state is the energetic ground state at low temperatures.

In bulk and in solution the SCO phenomenon has been extensively studied since the 60s [6]. To exploit the spin-switching functionality for a future spin electronic on the molecular level, the complexes have to be contacted and immobilized, which requires to bring them into contact with a solid surface. However, the additional interaction with a surface acts on the metastable balance of the spin states and easily results in a quenching of the SCO transition.

The vast majority of the known SCO molecules are salts which complicates the preparation of well-defined surface layers due to the presence of counter ions. Vacuum deposition is a way to obtain high quality surface layers, but requires sublimation of the complexes, which is typically not possible with salts. A solution are neutral SCO complexes in which the positive charge of the metal center is compensated by negative charges on the ligands. Only for a few number of such complexes successful vacuum deposition was reported [65, 66, 67, 68, 69, 70, 71, 72, 73, 74, 75].

$\left[\mathrm{Fe}(\mathrm{bpz})_{2}(\right.$ phen $\left.)\right]$, where $\mathrm{bpz}=\operatorname{dihydrobis}($ pyrazolyl)borate and phen $=1,10$ phenanthroline (inset of Fig. 10 (b)), is a neutral molecule that can be sublimated in vacuum at comparably low temperature of about $435 \mathrm{~K}$. Deposition on a $\mathrm{Au}(111)$ surface resulted in a fragmentation of the molecules into phen and $\left[\mathrm{Fe}(\mathrm{bpz})_{2}\right]$ for molecules in direct contact with the surface, as judged from x-ray absorption (XA) spectra [71]. This was confirmed by scanning tunneling microscopy (STM) images where phen dimers and four-coordinate Fe complexes could be identified [71]. No spin transition could be observed at submonolayer coverages, as can be seen from the absence of changes in Fe $L_{3}$ spectra with temperature shown in Fig. 9 (b). Only at higher coverages, like at 1.6 ML shown in Fig. 9 (a), a partial thermally induced spin transition can be observed. The amount of spin switching is compatible with the notion that only molecules in the second monolayer switch.

A study of the similar molecule $\left[\mathrm{Fe}(\mathrm{bpz})_{2}\right.$ (bipy)]/ $\mathrm{Au}(111)$ (bipy $=2,2$ '-bipyridine), in contrast, reported a spin transition of isolated complexes for $20 \%$ of the molecules at a coverage of 0.03-0.14 ML [72]. This apparent contradiction in the two reports disappears when looking at the peak-to-background intensity of the Fe spectra. As shown in the supporting information of Ref. [72], the Fe $L_{3}$ peak-to-background intensity, and thus the areal density of Fe ions, is comparable to the one of the 1.6 ML sample in Ref. [71]. Since both preparations are on the same substrate, the peak-to-background intensity is directly comparable. A likely cause of the discrepancy in the interpretation of the 


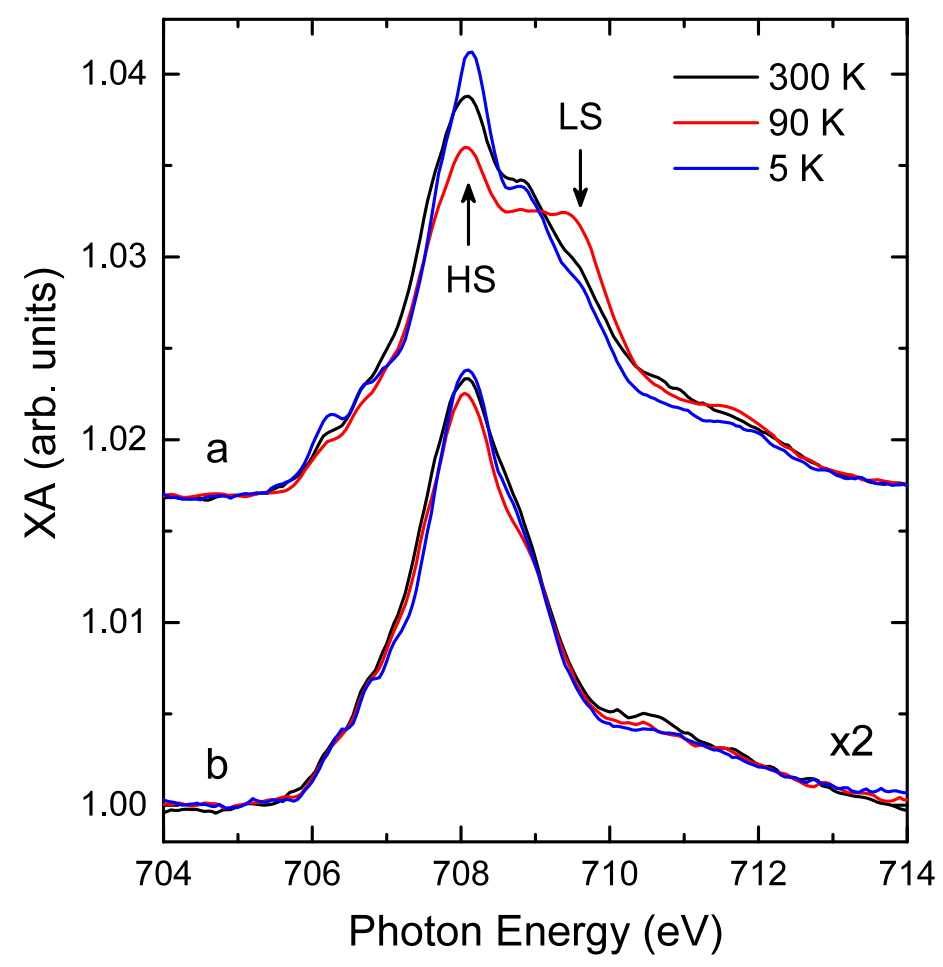

Figure 9. X-ray absorption spectra at the Fe $L_{3}$ edge recorded at an angle of incidence of $54.7^{\circ}$ at 300,90 , and $5 \mathrm{~K}$ of (a) $1.6 \mathrm{ML}$ and (b) $0.8 \mathrm{ML}$ of [Fe(bpz) $)_{2}$ (phen)] on $\mathrm{Au}(111)$. Arrows indicate the energies representative of molecules in the high-spin and low-spin states. While at $0.8 \mathrm{ML}$ coverage the spectra do not change with temperature, at the coverage of 1.6 ML some changes characteristic for a thermal transition from a high-spin state at $300 \mathrm{~K}$ towards an increasing content of low-spin state at lower temperatures is observed. After [71], copyright 2013 Wiley-VCH Verlag GmbH \& Co.

coverage in Refs. [71] and [72], 1.6 vs. $0.03-0.14 \mathrm{ML}$, could be that in the latter the peak-to-background intensity has been compared to the one of a thick film of the same molecules, in which no signal from the substrate is present, without considering that the x-ray absorption at the Fe $L_{3}$ pre-edge (around $705 \mathrm{eV}$ ) of $\mathrm{Au}$, which dominates the background intensity at small coverages, is more than one order of magnitude higher than that of the molecular film, which mainly consists of carbon [76]. Considering that the coverages are similar, the two similar molecules thus also behave similarly with respect to thermal SCO switching. Relying on the coverage dependence of the switching and the additional STM work of Ref. [71], this observed thermal SCO switching most likely has to be ascribed in both cases to molecules not in direct contact with the $\mathrm{Au}$ substrate and a coverage higher than one monolayer.

Changing the substrate to highly ordered pyrolytic graphite (HOPG) as a conductive and even more weakly interacting substrate compared to $\mathrm{Au}$, a complete thermal spin transition of vacuum-deposited [Fe(NCS) $\left.)_{2} \mathrm{~L}\right](\mathrm{L}=1-\{6-[1,1-d i$ (pyridin-2yl)ethyl]-pyridin-2-yl\}- $N, N$-dimethylmethanamine) [67] and [Fe(bpz $)_{2}$ (phen)] molecules [77] could be observed at submonolayer coverages. Formation of three-dimensional crystallites was excluded in both cases by means of low-energy electron diffraction and 

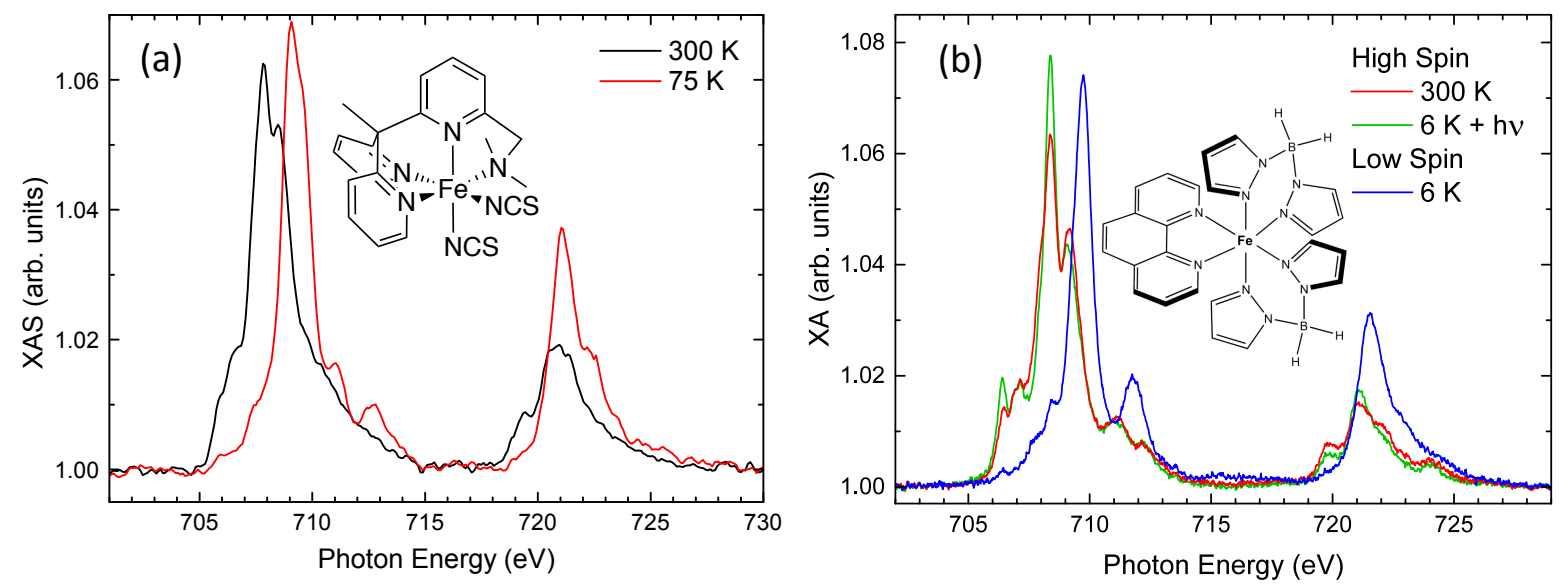

Figure 10. Temperature-dependent $\mathrm{Fe} L_{2,3} \mathrm{XA}$ spectra recorded at an angle of incidence of $54.7^{\circ}$. (a) Spectra taken at $300 \mathrm{~K}$ (black) and $75 \mathrm{~K}$ (red) of $0.8 \mathrm{ML}$ of $\left[\mathrm{Fe}(\mathrm{NCS})_{2} \mathrm{~L}\right]$ on highly ordered pyrolytic graphite. Reprinted with permission from [67]. Copyright 2012 American Chemical Society. (b) Spectra taken at $300 \mathrm{~K}$ (red) and $6 \mathrm{~K}$ (blue) of $0.4 \mathrm{ML}$ of $\left[\mathrm{Fe}(\mathrm{bpz})_{2}\right.$ (phen)] on highly ordered pyrolytic graphite, as well as a spectrum taken at $6 \mathrm{~K}$ after illumination with green light (green). After [77]. Copyright 2015 American Chemical Society. In both cases, the spectral shape changes completely when going from room temperature to low temperature, evidencing a nearcomplete thermal spin transition between high spin and low spin. The illumination with green light in (b) induces the transition back to the high-spin state with a spectrum closely resembling the one recorded at room temperature. The insets show the chemical structure of the respective molecules.

atomic force microscopy, respectively. An Fe $L_{2,3}$ spectrum of $\left[\mathrm{Fe}(\mathrm{NCS})_{2} \mathrm{~L}\right]$ (inset in Fig. 10 (a)) on HOPG at room temperature is shown by the black line in Fig. 10 (a). It displays two main $L_{3}$ resonances at 707.8 and $708.5 \mathrm{eV}$, reflecting the splitting of the $e_{g}$ and $t_{2 g}$ empty density of states in the high-spin state. At $75 \mathrm{~K}$, the shape of the Fe spectrum (blue line) is completely modified displaying a single Fe $L_{3}$ resonance at $709.1 \mathrm{eV}$, which can be assigned to the $e_{g}$ empty density of states of the low-spin state. For a realistic description of the XA spectrum the multi-electron nature of the electronic states has to be taken into account giving rise to additional fine structure in the spectra as seen in Fig. 10, which can be calculated by means of multiplet theory. Anyway, the very small intensity of the low-spin spectrum (red curve) at the energies at which the high-spin spectrum has high intensity shows that the conversion of the adsorbed molecules is virtually complete. A more detailed comparison of the two spectra and deconvolution into spectra assigned to the "pure" high-spin and low-spin states reveals that at $75 \mathrm{~K}$ all of the molecules are in the low-spin state, while at $300 \mathrm{~K}$ about $90 \%$ are in the high-spin state [67].

The temperature-dependent spectra of $0.4 \mathrm{ML}$ of $\left[\mathrm{Fe}(\mathrm{bpz})_{2}(\mathrm{phen})\right]$ on $\mathrm{HOPG}$ are qualitatively identical. Also here the spectrum taken at room temperature (red line in Fig. 10 (b)) exhibits a double-peak structure, shifted by $0.6 \mathrm{eV}$ to higher photon energies compared to the case of $\left[\mathrm{Fe}(\mathrm{NCS})_{2} \mathrm{~L}\right]$ molecules. The spectrum at $6 \mathrm{~K}$ (blue line) shows 
a single peak at higher energy, evidencing the complete conversion of the molecules into the low-spin state [77].

While this spin-state switching of surface-anchored molecules by temperature variation is certainly very interesting, switching at constant temperature would be more relevant for applications. The so-called "light-induced excited spin-state trapping" (LIESST), which has been observed for certain SCO molecules in bulk material $[78,79,7,80]$, might be a way to accomplish that. LIESST means the optical pumping of the metal ion at low temperatures at which the low-spin state is the ground state into the metastable high-spin state. The pumping mechanism is based on an excitation of the metal-to-ligand charge transfer band whereby an electron is promoted from the Fe ion to one of the ligands. This excited state decays fast to the high-spin state with a quantum yield close to unity [81]. If the temperature does not exceed the temperature needed to thermally overcome the barrier separating the metastable high-spin from the energetically lower-lying low-spin state, the high-spin state reached after optical excitation is trapped, hence the name.

This LIESST effect could also be observed in $\left[\mathrm{Fe}(\mathrm{bpz})_{2}\right.$ (phen)] molecules adsorbed on HOPG. Illumination of a submonolayer of molecules at $6 \mathrm{~K}$ with green light leads to a complete change of the Fe $L_{2,3}$ spectrum (green line in Fig. 10 (b)), evidencing a full LIESST transition [71]. This light-induced transition of molecules in direct contact with a solid surface is highly efficient with an effective cross section being lower by only one order of magnitude compared to bulk samples [82]. It thus provides optical control over the magnetic moment of the adsorbed complexes. A strategy to obtain lightinduced spin-state switching at room temperature is to use photoisomerizable molecules as ligands, which has been demonstrated in the solid phase for an Fe complex containing a diarylethene ligand [83].

Vacuum-deposited SCO molecules have been also studied by means of STM. $\left[\mathrm{Fe}(\mathrm{bpz})_{2}\right.$ phen $]$ molecules of the second molecular layer on $\mathrm{Au}(111)$, sitting on a complete layer of decomposed $\left[\mathrm{Fe}(\mathrm{bpz})_{2}\right]$ and (phen), could be distinguished with respect to their spin state by their different appearance in STM images. Individual molecules could then be switched in a controlled way from low spin to high spin by placing the tip above them and applying a voltage pulse [84]. The reverse switching, from high spin to low spin, was observed in a random way in a larger vicinity of the tip after tunneling a relatively high current between tip and sample [84]. Recently, light-induced switching of a fraction of a submonolayer of $\left[\mathrm{Fe}\left(\left(3,5-\left(\mathrm{CH}_{3}\right)_{2} \mathrm{pz}\right)_{3} \mathrm{BH}\right)_{2}\right](\mathrm{pz}=$ pyrazolyl $)$ on $\mathrm{Au}(111)$ has been reported and the propagation of the excited phase has been monitored using STM [74].

In another STM study, isolated $\left[\mathrm{Fe}(\right.$ phen $\left.)(\mathrm{NCS})_{2}\right]$ complexes, decoupled from a $\mathrm{Cu}(001)$ surface by a copper nitride layer, have been investigated. Also here the two spin states could be distinguished by their different appearance in STM images [85]. In this system, a controlled deterministic switching by the STM tip has been reported. When the bias voltage between tip and sample exceeds a certain threshold voltage, switching of the spin state of the molecule underneath the tip is observed. The direction 


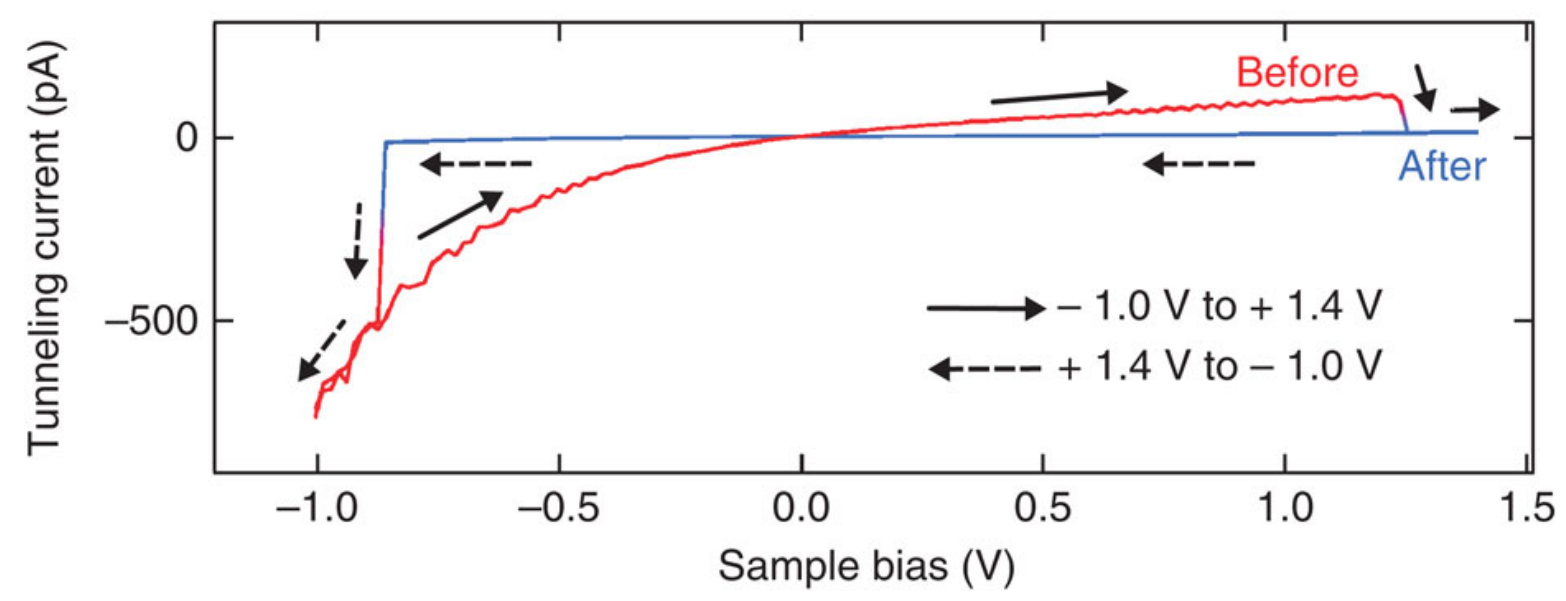

Figure 11. Scanning tunneling spectroscopy $I(V)$ curves recorded at the center of $\mathrm{Fe}(\text { phen })_{2}(\mathrm{NCS})_{2}$ molecules adsorbed on $\mathrm{CuN} / \mathrm{Cu}(001)$. The molecules can be switched between the high-spin (red lines) and low-spin state (blue lines) by applying high bias voltages between tip and sample. Together with the different tunneling characteristics this leads to a memristive behavior. Reprinted from [85] by permission from Macmillan Publishers Ltd: Nature Communications, copyright 2012.

of the switching hereby depends on the polarity of the tip voltage: Positive voltages only trigger the switching from high spin to low spin, while negative voltages only lead to the reverse switching from low spin to high spin [85]. Since the molecule exhibits a higher tunnel conductivity in the high-spin state compared to the low-spin state, this results in a memristive behavior. (A memristor is a resistor the resistance of which can be switched by the applied voltage, leading to bistability and hysteresis in the current-voltage curves.) Fig. 11 shows conductance $(I(V))$ curves of an individual $\left[\mathrm{Fe}(\right.$ phen $\left.)(\mathrm{NCS})_{2}\right]$ molecule on $\mathrm{CuN} / \mathrm{Cu}(001)$. The red curve is obtained for increasing the bias voltage while the molecule is in the high-spin state. At a voltage of about $+1.2 \mathrm{~V}$, switching to the low-spin state occurs. Reducing then the voltage, the blue curve is obtained, characteristic for the molecule in the low-spin state. At a negative voltage of about $-0.8 \mathrm{~V}$, switching to the high-spin state occurs. Both together leads to a hysteresis during cycles of voltage sweeps, as displayed in Fig. 11, provided each scan exceeds the threshold voltages for switching [85]. These findings illustrate the feasibility of spin electronics on the molecular level, in which not only the magnetic moment of an adsorbed molecule can be switched on and off, but also the spin state can be read out electronically.

\section{Outlook}

The presented examples have shown that the magnetism of adsorbed metal-organic molecules is an actual and rapidly emerging field of research. This topical review focussed only on systems in which the magnetic properties can be controlled by external means. By gaining control on the size or the direction of the magnetic moment of 
adsorbed molecules, certain functionalities are brought to the surface and, in a more general way, to nanoscopic building blocks of spin-electronic devices. If the creativity in designing new molecules with interesting properties by synthetic chemistry can be channelled to systems that can be deposited on a solid surface, exciting new possibilities for functional nanodevices can be foreseen.

However, as, in particular, the last example of the spin crossover molecules has shown, it is not straightforward to bring existing functionalities onto a surface. There are several examples of molecules that work well in solution, but might not work on a surface without adequate modification. One of these are hybrid molecules for lightinduced manipulation of the magnetic properties that contain a photochromic switch and a magnetic entity. Combining the photochromic switching capability of an azobenzene unit and the susceptibility of a metal porphyrin to additional coordination, like discussed in section 3 of this review, yields photoswitchable molecules that work perfectly in solution [5]. Similarly, spin-crossover molecules with a photoswitchable diarylethen ligand show promising light-induced control on the magnetic properties [86]. However, it is a challenge to bring these molecular functionalities to the surface. To achieve this, it might be necessary to redesign the molecules specifically for functioning in the adsorbed state. In this sense, it is also a challenge for synthetic chemistry to create molecules that exhibit such interesting properties on surfaces.

There are strategies to bring certain functionalities to the surface. The role of the surface in this is threefold. In some cases it is only the platform to immobilize the functional molecules and to keep them in place while at the same time not disturbing their specific function. In that case the interaction should be strong enough to fix the molecule at a certain place, but not too strong in order not to interfere with its function. Highly oriented pyrolytic graphite, as in the last examples, would be such a substrate. In other cases the substrate is an essential part of the system, like the magnetic substrates discussed in section 2. Here the substrate participates in the magnetic coupling, it is thus essential for the functionality. In a third class of systems the main functionality is in the substrate, and magnetic molecules and their (possibly externally controllable magnetism) are the means to manipulate this functionality. An example are surfaces with nontrivial transport or topological properties such as topological insulators [87, 88]. Paramagnetic molecules might be an advantageous alternative means instead of metal atoms for magnetic surface doping of topological insulators, a lively discussed topics [89, 90, 91, 92]. Using molecules as a means to locally modify the electronic properties of such surfaces and in addition controlling the magnetism of these adsorbed molecules could provide a handle to switch such modifications on and off.

Considering the wealth of molecular function in solution, the possibilities to design magnetic metal-organic molecules, and the interest in functional surface structures and hybrid interfaces, we expect that the field is right now just in its infancy, and that we will witness a rapid growth in width and depth accompanied by an increasing number of fundamental breakthroughs. 


\subsection{Acknowledgments}

Financial support from the German Science Foundation (DFG) within the frame of the collaborative research center Sfb 658 ("Elementary processes in molecular switches at surfaces") is gratefully acknowledged. The authors thank G. Ahmadi, M. E. Ali, L. M. Arruda, K. Baberschke, A. Bannwarth, R. Berndt, P. W. Brouwer, Y.-M. Chang, P. Eckhold, O. Eriksson, K. J. Franke, T. G. Gopakumar, A. Grohmann, B. W. Heinrich, C. F. Hermanns, L. Kipgen, W. Kroener, A. Krüger, D. Krüger, B. Krumme, W. Kuch, J. Kurde, J. Luo, F. Matino, J. Miguel, S. Mühlenberend, P. Müller, H. Naggert, F. Nickel, P. M. Oppeneer, P. M. Panchmatia, M. Piantek, N. Ponpandian, D. Rolf, B. Sanyal, C. Schmidt, E. Schierle, M. L. Schneider, P. Srivastava, C. Sorg, K. Tarafder, F. Tuczek, W. Walter, S. T. Waßerroth, H. Wende, E. Weschke, D. Wiedemann, and

$\mathrm{X}$. $\mathrm{Xu}$ for their involvement in various parts of the reported research.

[1] S. A. Wolf, D. D. Awschalom, R. A. Buhrman, J. M. Daughton, S. von Molnár, M. L. Roukes, A. Y. Chtchelkanova, and D. M. Treger. Spintronics: A spin-based electronics vision for the future. Science, 294:1488-1495, 2001.

[2] D. D. Awschalom and M. E. Flatté. Challenges for semiconductor spintronics. Nat. Phys., 3:153$159,2007$.

[3] S. Bandyopadhyay and M. Cahay. Introduction to Spintronics. CRC Press, Boca Raton London New York, 2016.

[4] E. Y. Zymbal and I. Žutić, editors. Handbook of Spin Transport and Magnetism. CRC Press, Boca Raton, London, New York, 2011.

[5] S. Venkataramani, U. Jana, M. Dommaschk, F. D. Sönnichsen, F. Tuczek, and R. Herges. Magnetic bistabllity of molecules in homogeneous solution at room temperature. Science, 331:445-448, 2011.

[6] P. Gütlich and H. A. Goodwin, editors. Spin Crossover in Transition Metal Compounds I. Springer, Berlin, 2004.

[7] P. Gütlich, A. B. Gaspar, and Y. Garcia. Spin state switching in iron coordination compounds. Beilstein J. Org. Chem., 9:342-391, 2014.

[8] K. M. Kadish, K. M. Smith, and R. Guilard, editors. Handbook of Porphyrin Science, volume 2. World Scientific, New Jersey, London, Singapore, Beijing, Shanghai, Hong Kong, Taipei, Chennai, 2010.

[9] W. Auwärter, D. Écija, F. Klappenberger, and J. V. Barth. Porphyrins at interfaces. Nat. Chem., 7:105-120, 2015.

[10] J. M. Gottfried. Surface chemistry of porphyrins and phthalocyanines. Surf. Sci. Rep., 70:259-379, 2015.

[11] H. Peisert, J. Uihlein, F. Petraki, and Th. Chassé. Charge transfer between transition metal phthalocyanines and metal substrates: The role of the transition metal. J. Electron Spectrosc. Relat. Phenom., 204:49-60, 2015.

[12] A. Scheybal, T. Ramsvik, R. Bertschinger, M. Putero, F. Nolting, and T. A. Jung. Induced magnetic ordering in a molecular monolayer. Chem. Phys. Lett., 411:214-220, 2005.

[13] H. Wende, M. Bernien, J. Luo, C. Sorg, N. Ponpandian, J. Kurde, J. Miguel, M. Piantek, X. Xu, P. Eckhold, W. Kuch, K. Baberschke, P. M. Panchmatia, B. Sanyal, P. M. Oppeneer, and O. Eriksson. Substrate-induced magnetic ordering and switching of iron porphyrin molecules. Nat. Mater., 6:516-520, 2007.

[14] J. Stöhr and H. C. Siegmann, editors. Magnetism: From Fundamentals to Nanoscale Dynamics. Springer, Berlin, 2006. 
[15] A. Lodi Rizzini, C. Krull, T. Balashov, J. J. Kavich, A. Mugarza, P. S. Miedema, P. K. Thakur, V. Sessi, S. Klyatskaya, M. Ruben, S. Stepanow, and P. Gambardella. Coupling single molecule magnets to ferromagnetic substrates. Phys. Rev. Lett., 107:177205, 2011.

[16] M. Bernien, J. Miguel, C. Weis, M. E. Ali, J. Kurde, B. Krumme, P. M. Panchmatia, B. Sanyal, M. Piantek, P. Srivastava, K. Baberschke, P. M. Oppeneer, O. Eriksson, W. Kuch, and H. Wende. Tailoring the nature of magnetic coupling of Fe-porphyrin molecules to ferromagnetic substrates. Phys. Rev. Lett., 102:047202, 2009.

[17] S. Javaid, M. Bowen, S. Boukari, L. Joly, J.-B. Beaufrand, Xi Chen, Y. J. Dappe, F. Scheurer, J.-P. Kappler, J. Arabski, W. Wulfhekel, M. Alouani, and E. Beaurepaire. Impact on interface spin polarization of molecular bonding to metallic surfaces. Phys. Rev. Lett., 105:077201, 2010.

[18] C. Wäckerlin, D. Chylarecka, A. Kleibert, K. Müller, C. Iacovita, F. Nolting, T. A. Jung, and N. Ballav. Controlling spins in adsorbed molecules by a chemical switch. Nat. Commun., 1:61:doi: 10.1038/ncomms1057, 2010.

[19] D. Chylarecka, T. K. Kim, K. Tarafder, K. Müller, K. Gödel, I. Czekaj, C. Wäckerlin, M. Cinchetti, M. E. Ali, C. Piamonteze, F. Schmitt, J.-P. Wüstenberg, C. Ziegler, F. Nolting, M. Aeschlimann, P. M. Oppeneer, N. Ballav, and T. A. Jung. Indirect magnetic coupling of manganese porphyrin to a ferromagnetic cobalt substrate. J. Phys. Chem. C, 115:1295-1301, 2011.

[20] S. Bhandary, B. Brena, P. M. Panchmatia, I. Brumboiu, M. Bernien, C. Weis, B. Krumme, C. Etz, W. Kuch, H. Wende, O. Eriksson, and B. Sanyal. Manipulation of spin state of iron porphyrin by chemisorption on magnetic substrates. Phys. Rev. B, 88:024401, 2013.

[21] N. Ishikawa, M. Sugita, T. Ishikawa, S.-y. Koshihara, and Y. Kaizu. Lanthanide double-decker complexes functioning as magnets at the single-molecular level. J. Am. Chem. Soc., 125:8694$8695,2003$.

[22] D. Klar, A. Candini, L. Joly, S. Klyatskaya, B. Krumme, P. Ohresser, J.-P. Kappler, M. Ruben, and $\mathrm{H}$. Wende. Hysteretic behaviour in a vacuum deposited submonolayer of single ion magnets. Dalton Trans., 43:10686-10689, 2014.

[23] D. Klar, S. Klyatskaya, A. Candini, B. Krumme, K. Kummer, Ph. Ohresser, V. Corradini, V. de Renzi, R. Biagi, L. Joly, J.-P. Kappler, U. del Pennino, M. Affronte, H. Wende, and M. Ruben. Antiferromagnetic coupling of $\mathrm{TbPc}_{2}$ molecules to ultrathin $\mathrm{Ni}$ and Co films. Beilstein J. Nanotech., 4:320-324, 2013.

[24] C. Nistor, C. Krull, A. Mugarza, S. Stepanow, C. Stamm, M. Soares, S. Klyatskaya, M. Ruben, and P. Gambardella. Exchange bias of $\mathrm{TbPc}_{2}$ molecular magnets on antiferromagnetic FeMn and ferromagnetic Fe films. Phys. Rev. B, 92:184402, 2015.

[25] A. Candini, D. Klar, S. Marocchi, V. Corradini, R. Biagi, V. De Renzi, U. del Pennino, F. Troiani, V. Bellini, S. Klyatskaya, M. Ruben, K. Kummer, N. B. Brookes, H. Huang, A. Soncini, H. Wende, and M. Affronte. Spin-communication channels between Ln(III) bis-phthalocyanines molecular nanomagnets and a magnetic substrate. Sci. Rep., 6:21740, 2016.

[26] A. Lodi Rizzini, C. Krull, A. Mugarza, T. Balashov, C. Nistor, R. Piquerel, S. Klyatskaya, M. Ruben, P. M. Sheverdyaeva, P. Moras, C. Carbone, Ch. Stamm, P. S. Miedema, P. K. Thakur, V. Sessi, M. Soares, F. Yakhou-Harris, J. C. Cezar, S. Stepanow, and P. Gambardella. Coupling of single, double, and triple-decker metal-phthalocyanine complexes to ferromagnetic and antiferromagnetic substrates. Surf. Sci., 630:361-374, 2014.

[27] J. Girovsky, K. Tarafder, Ch. Wäckerlin, J. Nowakowski, D. Siewert, T. Hählen, A. Wäckerlin, A. Kleibert, N. Ballav, Th. A. Jung, and P. M. Oppeneer. Antiferromagnetic coupling of Crporphyrin to a bare Co substrate. Phys. Rev. B, 90:220404, 2014.

[28] Ch. Wäckerlin, F. Donati, A. Singha, R. Baltic, A.-Ch. Uldry, B. Delley, S. Rusponi, and J. Dreiser. Strong antiferromagnetic exchange between manganese phthalocyanine and ferromagnetic europium oxide. Chem. Comm., 51:12958-12961, 2015.

[29] J. Klanke, E. Rentschler, K. Medjanik, D. Kutnyakhov, G. Schönhense, S. Krasnikov, I. V. Shvets, S. Schuppler, P. Nagel, M. Merz, and H. J. Elmers. Beyond the Heisenberg model: Anisotropic exchange interaction between a Cu-tetraazaporphyrin monolayer and $\mathrm{Fe}_{3} \mathrm{O}_{4}(100)$. Phys. Rev. 
Lett., 110:137202, 2013.

[30] R. Nünthel, T. Gleitsmann, P. Poulopoulos, A. Scherz, J. Lindner, E. Kosubek, Ch. Litwinski, Z. Li, H. Wende, K. Baberschke, S. Stolbov, and T. S. Rahman. Epitaxial growth of Ni on $\mathrm{Cu}(001)$ with the assistance of O-surfactant and its magnetism compared to $\mathrm{Ni} / \mathrm{Cu}(001)$. Surf. Sci., 531:53-67, 2003.

[31] C. Sorg, N. Ponpandian, M. Bernien, K. Baberschke, H. Wende, and R. Q. Wu. Induced magnetism of oxygen in surfactant-grown Fe, Co, and Ni monolayers. Phys. Rev. B, 73:064409, 2006.

[32] K. S. Novoselov, A. K. Geim, S. V. Morozov, D. Jiang, Y. Zhang, S. V. Dubonos, I. V. Grigorieva, and A. A. Firsov. Electric field effect in atomically thin carbon films. Science, 306:666-669, 2004.

[33] C. F. Hermanns, K. Tarafder, M. Bernien, A. Krüger, Y.-M. Chang, P. M. Oppeneer, and W. Kuch. Magnetic coupling of porphyrin molecules through graphene. Adv. Mater., 25:3473-3477, 2013.

[34] Yu. S. Dedkov, M. Fonin, U. Rüdiger, and C. Laubschat. Rashba effect in the graphene/Ni(111) system. Phys. Rev. Lett., 100:107602, 2008.

[35] A. Varykhalov, J. Sánchez-Barriga, A. M. Shikin, C. Biswas, E. Vescovo, A. Rybkin, D. Marchenko, and O. Rader. Electronic and magnetic properties of quasifreestanding graphene on Ni. Phys. Rev. Lett., 101:157601, 2008.

[36] M. Bernien, X. Xu, J. Miguel, M. Piantek, P. Eckhold, J. Luo, J. Kurde, W. Kuch, K. Baberschke, H. Wende, and P. Srivastava. Fe-porphyrin monolayers on ferromagnetic substrates: Electronic structure and magnetic coupling strength. Phys. Rev. B, 76:214406, 2007.

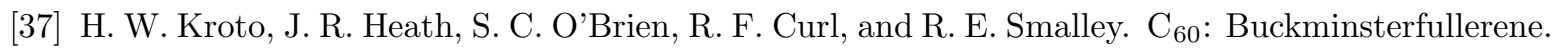
Nature, 318:162-163, 1985.

[38] H. Ehrenreich and F. Spaepen, editors. Solid State Physics, volume 48. Academic Press, Boston, 1994.

[39] D. S. Bethune, R. D. Johnson, J. R. Salem, M. S. de Vries, and C. S. Yannoni. Atoms in carbon cages: the structure and properties of endohedral fullerenes. Nature, 366:123-128, 1993.

[40] R. Westerström, J. Dreiser, C. Piamonteze, M. Muntwiler, S. Weyeneth, H. Brune, S. Rusponi, F. Nolting, A. Popov, S. Yang, L. Dunsch, and T. Greber. An endohedral single-molecule magnet with long relaxation times: $\mathrm{DySc}_{2} \mathrm{~N} @ \mathrm{C}_{80}$. J. Am. Chem. Soc., 134:9840, 2012.

[41] C. F. Hermanns, M. Bernien, A. Krüger, C. Schmidt, S. T. Waßerroth, G. Ahmadi, B. W. Heinrich, M. L. Schneider, P. W. Brouwer, K. J. Franke, E. Weschke, and W. Kuch. Magnetic coupling of $\mathrm{Gd}_{3} \mathrm{~N} @ \mathrm{C}_{80}$ endohedral fullerenes to a substrate. Phys. Rev. Lett., 111:167293, 2013.

[42] M. Gruber, F. Ibrahim, S. Boukari, L. Joly, V. Da Costa, M. Studniarek, M. Peter, H. Isshiki, H. Jabbar, V. Davesne, J. Arabski, E. Otero, F. Choueikani, K. Chen, P. Ohresser, W. Wulfhekel, F. Scheurer, E. Beaurepaire, M. Alouani, W. Weber, and M. Bowen. Spin-dependent hybridization between molecule and metal at room temperature through interlayer exchange coupling. Nano Lett., 15:7921-7926, 2015.

[43] J. E. Ortega and F. J. Himpsel. Quantum well states as mediators of magnetic coupling in superlattices. Phys. Rev. Lett., 69:844-847, 1992.

[44] C. Carbone, E. Vescovo, O. Rader, W. Gudat, and W. Eberhardt. Exchange split quantum well states of a noble metal film on a magnetic substrate. Phys. Rev. Lett., 71:2805-2808, 1993.

[45] R. Kläsges, D. Schmitz, C. Carbone, W. Eberhardt, P. Lang, R. Zeller, and P. H. Dederichs. Shortperiod oscillations in photoemission from $\mathrm{Cu}$ films on $\mathrm{Co}(100)$. Phys. Rev. B, 57:R696-R699, 1998.

[46] B. Heinrich and J. A. C. Bland, editors. Ultrathin Magnetic Structures II. Springer, Berlin, 1994.

[47] M. D. Stiles. Interlayer exchange coupling. J. Magn. Magn. Mater., 200:322, 1999.

[48] P. Grünberg, R. Schreiber, Y. Pang, M. B. Brodsky, and H. Sowers. Layered magnetic structures: Evidence for antiferromagnetic coupling of Fe layers across Cr interlayers. Phys. Rev. Lett., 57:2442-2445, 1986.

[49] S. S. P. Parkin, N. More, and K. P. Roche. Oscillations in exchange coupling and magnetoresistance in metallic superlattice structures: Co/Ru, Co/Cr, and Fe/Cr. Phys. Rev. Lett., 64:2304-2307, 
1990.

[50] S. S. P. Parkin. Systematic variation of the strength and oscillation period of indirect magnetic exchange coupling through the 3d, 4d, and 5d transition metals. Phys. Rev. Lett., 67:3598, 1991.

[51] P. Bruno and C. Chappert. Oscillatory coupling between ferromagnetic layers separated by a nonmagnetic metal spacer. Phys. Rev. Lett., 67:1602-1605, 1991.

[52] D. M. Edwards, J. Mathon, R. B. Muniz, and M. S. Phan. Oscillations of the exchange in magnetic multilayers as an analog of de Haas-van Alphen effect. Phys. Rev. Lett., 67:493-496, 1991.

[53] P. Bruno. Theory of interlayer magnetic coupling. Phys. Rev. B, 52:411-439, 1995.

[54] A. Lodi Rizzini, C. Krull, T. Balashov, A. Mugarza, C. Nistor, F. Yakhou, V. Sessi, S. Klyatskaya, M. Ruben, S. Stepanow, and P. Gambardella. Exchange biasing single molecule magnets: Coupling of $\mathrm{TbPc}_{2}$ to antiferromagnetic layers. Nano Letters, 12:5703-5707, 2012.

[55] J. Nogués and I. K. Schuller. Exchange bias. J. Magn. Magn. Mater., 192:203-232, 1999.

[56] F. Radu and H. Zabel. Exchange bias effect of ferro-/antiferromagnetic heterostructures. In H. Zabel and S. D. Bader, editors, Magnetic Heterostructures, volume 227, pages 97-184. Springer, Berlin Heidelberg New York, 2008.

[57] S. Gueddida, M. Gruber, T. Miyamachi, E. Beaurepaire, W. Wulfhekel, and M. Alouani. Exchange coupling of spin-crossover molecules to ferromagnetic Co islands. J. Phys. Chem. Lett., 7:900904, 2016.

[58] K. Flechtner, A. Kretschmann, H.-P. Steinrück, and J. M. Gottfried. NO-induced reversible switching of the electronic interaction between a porphyrin-coordinated cobalt ion and a silver surface. J. Am. Chem. Soc., 129:12110-12111, 2007.

[59] J. Miguel, C. F. Hermanns, M. Bernien, A. Krüger, and W. Kuch. Reversible manipulation of the magnetic coupling of single molecular spins in Fe-porphyrins to a ferromagnetic substrate. $J$. Phys. Chem. Lett., 2:1455-1459, 2011.

[60] C. F. Hermanns, M. Bernien, A. Krüger, J. Miguel, and W. Kuch. Switching the electronic properties of Co-octaethylporphyrin molecules on oxygen-covered Ni films by NO adsorption. J. Phys.: Cond. Matt., 24:394008, 2012.

[61] A. Stróżecka, M. Soriano, J. I. Pascual, and J. J. Palacios. Reversible change of the spin state in a manganese phthalocyanine by coordination of CO molecule. Phys. Rev. Lett., 109:147202, Oct 2012 .

[62] C. Wäckerlin, K. Tarafder, D. Siewert, J. Girovsky, T. Hählen, C. Iacovita, A. Kleibert, F. Nolting, T. A. Jung, P. M. Oppeneer, and N. Ballav. On-surface coordination chemistry of planar molecular spin systems: Novel magnetochemical effects induced by axial ligands. Chem. Sci., 3:3154-3160, 2012.

[63] C. Wäckerlin, K. Tarafder, J. Girovsky, J. Nowakowski, T. Hählen, A. Shchyrba, D. Siewert, A. Kleibert, F. Nolting, P. M. Oppeneer, T. A. Jung, and N. Ballav. Ammonia coordination introducing a magnetic moment in an on-surface low-spin porphyrin. Angew. Chem. Int. Ed., 52:4568-4571, 2013.

[64] C. Wäckerlin, J. Nowakowski, S.-X. Liu, M. Jaggi, D. Siewert, J. Girovsky, A. Shchyrba, T. Hählen, A. Kleibert, P. M. Oppeneer, F. Nolting, S. Decurtius, T. A. Jung, and N. Ballav. Twodimensional supramolecular electron spin arrays. Adv. Mater., 25:2404-2408, 2013.

[65] H. Naggert, A. Bannwarth, S. Chemnitz, T. von Hofe, E. Quandt, and F. Tuczek. First observation of light-induced spin change in vacuum deposited thin films of iron spin crossover complexes. Dalton Trans., 40:6364-6366, 2011.

[66] T. Mahfoud, G. Molnár, S. Cobo, L. Salmon, C. Thibault, C. Vieu, P. Demont, and A. Bousseksou. Electrical properties and non-volatile memory effect of the $\left[\mathrm{Fe}\left(\mathrm{HB}(\mathrm{pz})_{3}\right)_{2}\right]$ spin crossover complex integrated in a microelectrode device. Appl. Phys. Lett., 99:053307, 2011.

[67] M. Bernien, D. Wiedemann, C. F. Hermanns, A. Krüger, D. Rolf, W. Kroener, P. Müller, A. Grohmann, and W. Kuch. Spin crossover in a vacuum-deposited submonolayer of a molecular iron(II) complex. J. Phys. Chem. Lett., 3:3431-3434, 2012.

[68] T. Palamarciuc, J. C. Oberg, F. El Hallak, C. F. Hirjibehedin, M. Serri, S. Heutz, J.-F. Létard, and 
P. Rosa. Spin crossover materials evaporated under clean high vacuum and ultra-high vacuum conditions: from thin films to single molecules. J. Mater. Chem., 22:9690-9695, 2012.

[69] E. C. Ellingsworth, B. Turner, and G. Szulczewski. Thermal conversion of $\left[\mathrm{Fe}(\mathrm{phen})_{3}\right](\mathrm{SCN})_{2}$ thin films into the spin crossover complex Fe(phen $)_{2}(\mathrm{NCS})_{2}$. RSC Adv., 3(11):3745, 2013.

[70] B. Schäfer, C. Rajnák, I. Šalitroš, O. Fuhr, D. Klar, C. Schmitz-Antoniak, E. Weschke, H. Wende, and M. Ruben. Room temperature switching of a neutral molecular iron(II) complex. Chem. Commun., 49:10986-10988, 2013.

[71] T. G. Gopakumar, M. Bernien, H. Naggert, F. Matino, C. F. Hermanns, A. Bannwarth, S. Mühlenberend, A. Krüger, D. Krüger, F. Nickel, W. Walter, R. Berndt, W. Kuch, and F. Tuczek. Spin-crossover complex on $\mathrm{Au}(111)$ : Structural and electronic differences between mono- and multilayers. Chem. Eur. J., 19:15702-15709, 2013.

[72] B. Warner, J. C. Oberg, T. G. Gill, F. El Hallak, C. F. Hirjibehedin, M. Serri, S. Heutz, M.-A. Arrio, P. Sainctavit, M. Mannini, G. Ponetti, R. Sessoli, and P. Rosa. Temperature- and lightinduced spin crossover observed by x-ray spectroscopy on isolated Fe(II) complexes on gold. $J$. Phys. Chem. Lett., 4:1546-1552, 2013.

[73] H. Naggert, J. Rudnik, L. Kipgen, M. Bernien, F. Nickel, L. M. Arruda, W. Kuch, C. Näther, and F. Tuczek. Vacuum-evaporable spin-crossover complexes: physicochemical properties in the crystalline bulk and in thin films deposited from the gas phase. J. Mater. Chem. C, 3:7870-7877, 2015.

[74] K. Bairagi, O. Iasco, A. Bellec, A. Kartsev, Dongzhe Li, J. Lagoute, C. Chacon, Y. Girard, S. Rousset, F. Miserque, Y. J. Dappe, A. Smogunov, C. Barreteau, M.-L. Boillot, T. Mallah, and V. Repain. Molecular-scale dynamics of light-induced spin cross-over in a two-dimensional layer. Nat. Commun., 7:12212, 2016.

[75] S. Beniwal, X. Zhang, S. Mu, A. Naim, P. Rosa, G. Chastanet, J.-F. Létard, J. Liu, G. E. Sterbinsky, D. A. Arena, P. A. Dowben, and A. Enders. Surface-induced spin state locking of the $\left[\mathrm{Fe}\left(\mathrm{H}_{2} \mathrm{~B}(\mathrm{pz})_{2}\right)_{2}\right.$ (bipy)] spin crossover complex. J. Phys.: Cond. Matt., 28:206002, 2016.

[76] http://henke.lbl.gov/optical_constants/atten2.html.

[77] M. Bernien, H. Naggert, L. M. Arruda, L. Kipgen, F. Nickel, J. Miguel, C. F. Hermanns, A. Krüger, D. Krüger, E. Schierle, E. Weschke, F. Tuczek, and W. Kuch. Highly efficient thermal and lightinduced spin-state switching of an $\mathrm{Fe}(\mathrm{II})$ complex in direct contact with a solid surface. ACS Nano, 9:8960-8966, 2015.

[78] J. J. McGravey and I. Lawthers. Photochemically-induced perturbation of the ${ }^{1} \mathrm{~A} \rightleftharpoons{ }^{5} \mathrm{~T}$ equilibrium in $\mathrm{Fe}^{\mathrm{II}}$ complexes by pulsed laser irradiation in the metal-to-ligand charge-transfer absorption band. J. Chem. Soc., Chem. Commun., pages 906-907, 1982.

[79] S. Decurtins, P. Gütlich, C. P. Köhler, H. Spiering, and A. Hauser. Light-induced excited spin state trapping in a transition-metal complex: The hexa-1-propyltetrazole-iron (II) tetrafluoroborate spin-crossover system. Chem. Phys. Lett., 105:1 - 4, 1984.

[80] M. A. Halcrow, editor. Spin-Crossover Materials: Properties and Applications. John Wiley \& Sons, Ltd., New York, 2013.

[81] A. Hauser. Spin Crossover in Transition Metal Compounds II, chapter Light-Induced Spin Crossover and the High-Spin $\rightarrow$ Low-Spin Relaxation, pages 155-198. Springer Berlin Heidelberg, Berlin, Heidelberg, 2004.

[82] N. Moliner, L. Salmon, L. Capes, M. C. Muñoz, J.-F. Létard, A. Bousseksou, J.-P. Tuchagues, J. J. McGarvey, A. C. Dennis, M. Castro, R. Burriel, and J. A. Real. Thermal and optical switching of molecular spin states in the $\left\{\left[\mathrm{FeL}\left[\mathrm{H}_{2} \mathrm{~B}(\mathrm{pz})_{2}\right]_{2}\right\}\right.$ spin-crossover system $(\mathrm{L}=$ bpy, phen). $J$. Phys. Chem. B, 106:4276-4283, 2002.

[83] B. Rösner, M. Milek, A. Witt, B. Gobaut, P. Torelli, R. H. Fink, and M. M. Khusniyarov. Reversible photoswitching of a spin-crossover molecular complex in the solid state at room temperature. Angew. Chem. Int. Ed., 54:12976-12980, 2015.

[84] T. G. Gopakumar, F. Matino, H. Naggert, A. Bannwarth, F. Tuczek, and R. Berndt. Electroninduced spin crossover of single molecules in a bilayer on gold. Angew. Chem. Int. Ed., 51:6262- 
6266, 2012.

[85] T. Miyamachi, M. Gruber, V. Davesne, M. Bowen, S. Boukari, L. Joly, F. Scheurer, G. Rogez, T. K. Yamada, P. Ohresser, E. Beaurepaire, and W. Wulfhekel. Robust spin crossover and memristance across a single molecule. Nat. Commun., 3:938:doi: 10.1038/ncomms1940, 2012.

[86] M. Milek, F. W. Heinemann, and M. M. Khusniyarov. Spin crossover meets diarylethenes: Efficient photoswitching of magnetic properties in solution at room temperature. Inorg. Chem., 52:1158511592, 2013.

[87] M. Z. Hasan and C. L. Kane. Colloquium: Topological insulators. Rev. Mod. Phys., 82:3045-3067, 2010.

[88] J. E. Moore. The birth of topological insulators. Nature, 464:194-198, 2010.

[89] L. A. Wray, S.-Y. Xu, Y. Xia, D. Hsieh, A. V. Fedorov, Y. S. Hor, R. J. Cava, A. Bansil, H. Lin, and M. Z. Hasan. A topological insulator surface under strong Coulomb, magnetic and disorder perturbations. Nat. Phys., 7:32-37, 2011.

[90] T. Valla, Z.-H. Pan, D. Gardner, Y. S. Lee, and S. Chu. Photoemission spectroscopy of magnetic and nonmagnetic impurities on the surface of the $\mathrm{Bi}_{2} \mathrm{Se}_{3}$ topological insulator. Phys. Rev. Lett., 108:117601, 2012.

[91] M. R. Scholz, J. Sánchez-Barriga, D. Marchenko, A. Varykhalov, A. Volykhov, L. V. Yashina, and O. Rader. Tolerance of topological surface states towards magnetic moments: Fe on $\mathrm{Bi}_{2} \mathrm{Se}_{3}$. Phys. Rev. Lett., 108:256810, 2012.

[92] P. Sessi, F. Reis, T. Bathon, K. A. Kokh, O. E. Tereshchenko, and M. Bode. Signatures of Dirac fermion-mediated magnetic order. Nat. Commun., 5:5349:doi: 10.1038/ncomms6349, 2014. 